\title{
Regularity of traveling free surface water waves with vorticity
}

\author{
Hua Chen ${ }^{\mathrm{a}}$, Wei-Xi Li ${ }^{\mathrm{a}}$, Ling-Jun Wang ${ }^{\mathrm{b}}$ \\ ${ }^{a}$ School of Mathematics and Statistics, Wuhan University, 430072 Wuhan, China \\ ${ }^{b}$ School of Science, Wuhan University of Science and Technology, 430065 Wuhan, China
}

\begin{abstract}
We prove real analyticity of all the streamlines, including the free surface, of a gravity- or capillary-gravity-driven steady flow of water over a flat bed, with a Hölder continuous vorticity function, provided that the propagating speed of the wave on the free surface exceeds the horizontal fluid velocity throughout the flow. Furthermore, if the vorticity possesses some Gevrey regularity of index $s$, then the stream function admits the same Gevrey regularity throughout the fluid domain; in particular if the Gevrey index $s$ equals to 1 , then we obtain analyticity of the stream function. The regularity results hold for both periodic and solitary water waves.
\end{abstract}

Keywords: Analyticity, Gevrey regularity, wave profile, water wave, vorticity

\section{Introduction}

Recently, water waves with vorticity, also called rotational waves, are investigated extensively. There have been a series of works concerning rotational waves, including existence results for small- and large-amplitude waves [4, 6, 7, 11, 12, 21], as well as results on uniqueness and symmetry, analyticity of wave profile [3, 8, 10, 18, 19], and so on. The present work is mainly concerned with the analyticity or regularity results for rotational water waves, with or without surface tension.

Assuming that the vorticity function is Hölder continuously differentiable, Constantin and Strauss [4] proved, by using methods of bifurcation theory, the existence of global bifurcation branches consisting of periodic water waves which travel above a flat bottom with constant speed exceeding that of the water particles enclosed by the wave. The assumption that the wave speed exceeds that of the water particles is supported by field evidence [17], and means that the waves are not near breaking or stagnation. We consider such waves as well in this paper.

In the irrotational setting, a classical result due to Lewy [16] showed that irrotational waves without stagnation points have real analytic profiles, by use of a generalized Schwartz reflection principle. Recently, Constantin and Escher [3] generalized this result to rotational case, and proved that, under the same assumption on the vorticity function as in [4], namely Hölder continuity of the first derivative, each streamline, except the free

Email addresses: chenhua@whu.edu.cn (Hua Chen), wei-xi.li@whu.edu.cn (Wei-Xi Li), wanglingjun@wust.edu.cn (Ling-Jun Wang) 
surface, is real analytic; if further the vorticity function is real analytic, then the free surface itself is also analytic. The arguments in [3] base on translational invariance property of the resulting operator in the direction of wave propagation, and the celebrated result due to Kinderlehrer et al. [15] on regularity for elliptic free boundary problems. Later on, similar results as in [3] are obtained for deep-water waves [19], flows with merely bounded vorticity [18], solitary-water waves [14], and for periodic capillary-gravity waves [8, 9, 10] where it was shown that the wave profile is furthermore $C^{\infty}$-smooth if the vorticity function is Hölder continuously differentiable. Note that in the aforementioned works the analyticity of free surface is established under the extra assumption that the vorticity function is analytic.

It is natural to expect the analyticity of the free surface for flows with only Hölder continuous vorticity. This is what we will do in this work. Precisely, assuming that the vorticity function is only Hölder continuous, we obtain the real analyticity of all the streamlines, including the free surface, of the steady flow over a flat bed in the absence of stagnation points. As in the above works, we first use an appropriate hodograph change of variable that transforms the free boundary value problem (corresponding in a frame moving at the constant wave speed to the governing equations for water waves with vorticity) into a nonlinear boundary problem for a quasi-linear elliptic equation in a fixed rectangular domain. Then basing on some a priori Schauder estimates (see for instance [5, Theorem 6.30], and [1] for general nonlinear elliptic equations with nonlinear oblique boundary conditions), we show the analyticity of streamlines by giving successively a quantitative bound for each derivative of the streamlines in the Hölder norm.

We also study the case when the vorticity possesses more regularity property rather than Hölder continuity, namely Gevrey regularity of index $s$. Gevrey class is an intermediate space between the spaces of smooth functions and analytic functions, and the Gevrey class function of index 1 is just the real-analytic function; see Subsection 2.2 below for precise definition of Gevrey class. In this case we investigate Gevrey regularity of stream function throughout the fluid domain. If the vorticity is Gevrey regular, we prove that the stream function admits the same Gevrey regularity in the fluid domain, up to the free surface; see Theorem 2.4 stated in Subsection 2.2. To obtain this, we firstly establish the corresponding regularity for the height function in a fixed rectangular domain, and then use the result of [2, Theorem 3.1] to show that the Gevrey regularity is preserved through partial hodograph transformation.

We conclude this introduction by pointing out that our approach applies for both periodic and solitary waves. For simplicity we consider in this work only flows with finite depth. With suitable modifications, the methods may be employed to the periodic waves on deep water with vorticity, constructed in [11, 13].

This paper is organized as follows. In Section 2 we formulate the rotational waterwave problem as free boundary problem for stream function and its equivalent reformulation in a fixed rectangular domain, and state our main regularity results. Notations 
and some useful inequalities are listed. Section 3 is devoted to the proof of analyticity of streamlines including the free surface. In Section 4 we study the Gevrey (analytic) regularity of stream function. In the last section, Section 5, we consider the travelling capillary-gravity water waves, and obtain similar regularity results for streamlines and stream function.

\section{Preliminaries and main results}

\subsection{The governing equations for rotational water waves}

Consider a steady two-dimensional flow of an incompressible inviscid fluid over a rigid flat bed $y=-d$ with $0<d<\infty$, acted upon by gravity, and a steady wave on the free surface of the flow. By steady, we mean that the flow and the surface wave move at a constant speed from left to right without changing their configuration. In the frame of reference moving at the wave speed $c>0$, let the $x$-axis point in the direction of wave propagation, the free surface be given in the graph form by $y=\eta(x)$ and let the liquid occupy the stationary domain

$$
\Omega=\left\{(x, y) \in \mathbb{R}^{2}:-d<y<\eta(x)\right\}
$$

Take $y=0$ to represent the location of the undisturbed water surface. Let $(u(x, y), v(x, y))$ denote the velocity field, and define the stream function $\psi(x, y)$ by $\psi(x, \eta(x))=0$ and

$$
\psi_{y}=u-c, \quad \psi_{x}=-v
$$

The flow is allowed to be rotational and characterized by the vorticity $\omega=v_{x}-u_{y}$. Consider also only waves that are not near breaking or stagnation, so that

$$
\psi_{y}(x, y) \leq-\delta<0 \quad \text { in } \bar{\Omega}
$$

for some $\delta>0$, which implies that the vorticity $\omega$ is globally a function of the stream function $\psi$, denoted by $\gamma(-\psi)$; see [4]. The governing equations for the gravity water wave problem are formulated as

$$
\begin{array}{rc}
\triangle \psi=-\gamma(-\psi), & (x, y) \in \Omega, \\
|\nabla \psi|^{2}+2 g(y+d)=Q, & y=\eta(x), \\
\psi=0, & y=\eta(x), \\
\psi=-p_{0}, & y=-d .
\end{array}
$$

Here $g>0$ is the gravitational constant of acceleration, $Q$ is a constant related to the energy and

$$
p_{0}=\int_{-d}^{\eta(x)} \psi_{y}(x, y) d y<0
$$


is the relative mass flux (independent of $x$ ). Moreover the wave profile $\eta(x)$ represents an unknown in the problem since it is a free surface. We refer to [4] for the detailed derivation of the above system of governing equations.

The level sets $\{(x, y): \psi(x, y)=$ constant $\}$ are streamlines of the fluid motion. Note that the free surface and the rigid bottom are themselves streamlines in virtue of (3c) and $(3 \mathrm{~d})$. Observing (2), each streamline $\psi(x, y)=p$, with $p \in\left[p_{0}, 0\right]$, can be described by the graph of some function $y=\sigma_{p}(x)$.

\subsection{Statement of the main results}

To state our main results, we first recall the definition of Gevrey class functions, which is an intermediate space between the spaces of smooth functions and real-analytic functions; see [20, Chapter 1] for more detail.

Definition 2.1. Let $W$ be an open subset of $\mathbb{R}^{d}$ and $f$ be a real-valued function defined on the closure $\bar{W}$ of $W$. We say $f$ belongs to Gevrey class in $\bar{W}$ of index $s \geq 1$, denoted by $f \in G^{s}(\bar{W})$, if $f \in C^{\infty}(\bar{W})$ and for any compact subset $K$ of $\bar{W}$ there exists a constant $C_{K}$, depending only on $K$, such that

$$
\forall \alpha \in \mathbb{N}^{d}, \quad \max _{x \in K}\left|\partial^{\alpha} f(x)\right| \leq C_{K}^{|\alpha|+1}(|\alpha| !)^{s},
$$

where $|\alpha|=\alpha_{1}+\alpha_{2}+\cdots+\alpha_{d}$.

In particular $G^{1}(\bar{W})$ is the space of all real analytic functions in $\bar{W}$.

Throughout the paper let $C^{k, \mu}(\bar{W}), k \in \mathbb{N}, \mu \in(0,1)$, be the standard Hölder space of functions $f: \bar{W} \rightarrow \mathbb{R}$ with Hölder-continuous derivatives of exponent $\mu$ up to order $k$. For given $p_{0}<0$ and $\gamma \in C^{1, \mu}\left(\left[p_{0}, 0\right]\right)$, the existence of periodic and supercritical smallamplitude solitary water waves has been established in [4] and [7, 12], respectively. Our main result below shows that, with a Hölder continuous vorticity, each streamline can be described by the graph of some analytic function.

Theorem 2.2. Let the function $\gamma$ in (3a) belong to the Hölder space $C^{0, \mu}\left(\left[p_{0}, 0\right]\right)$ with $p_{0}<0$ and $0<\mu<1$ given, and let $\psi(x, y) \in C^{3, \mu}(\bar{\Omega})$ be the stream function for the boundary problem (3a)-(3d) with free surface $y=\eta(x)$. Suppose $\psi$ satisfies the no-stagnation assumption (2). Then each streamline including the free surface $y=\eta(x)$ is a real-analytic curve.

Remark 2.3. The existence of the stream function $\psi$ for the boundary problem (3ad)-(3d) is well-known (cf. [4] ).

The following result shows that the stream function admits the same regularity as the vorticity.

Theorem 2.4. Under the same assumptions as in Theorem [2.2, if $\gamma \in G^{s}\left(\left[p_{0}, 0\right]\right)$ additionally with $s \geq 1$ given, then we have $\psi(x, y) \in G^{s}(\bar{\Omega})$; in particular if $s=1$, i.e., $\gamma$ is analytic in $\left[p_{0}, 0\right]$, then the stream function $\psi(x, y)$ is analytic in $\bar{\Omega}$. 
Remark 2.5. The above results also hold for the travelling capillary-gravity water waves; see Theorem 5.1 in Section 5 .

\subsection{Reformulation}

Under the no-stagnation assumption (2), we can use the partial hodograph change of variables to transform the free boundary problem (3a)-(3d) into a problem with fixed boundary. Precisely, if we introduce the new variable $(q, p)$ with

$$
q=x, \quad p=-\psi(x, y),
$$

and exchange the roles of the $y$-coordinate and $\psi$ by setting

$$
h(q, p)=y+d,
$$

then the fluid domain $\Omega$ is transformed into a fixed infinite strip

$$
R=\left\{(q, p): q \in \mathbb{R}, p_{0}<p<0\right\},
$$

and the system (3a) $-(\underline{3 d})$ can be reformulated in this strip as

$$
\begin{aligned}
\left(1+h_{q}^{2}\right) h_{p p}-2 h_{p} h_{q} h_{p q}+h_{p}^{2} h_{q q}+\gamma(p) h_{p}^{3}=0, & \text { in } R, \\
1+h_{q}^{2}+(2 g h-Q) h_{p}^{2}=0, & \text { on } p=0, \\
h=0, & \text { on } p=p_{0} .
\end{aligned}
$$

We refer to [4] for the equivalence of the two systems (3a $)-(3 \mathrm{~d})$ and (4ad)-(4c) of governing equations. Note that $h_{p}=\frac{1}{c-u}$. The no-stagnation assumption (2) ensures that

$$
0<\inf _{(q, p) \in \bar{R}} h_{p} \leq h_{p} \leq \sup _{(q, p) \in \bar{R}} h_{p} \leq \frac{1}{\delta} .
$$

The following proposition shows that the regularity is preserved through hodograph transformation. So we only need to study the above problem (4a)-(4c) instead of the original one (3a) $-(3 \mathrm{~d})$.

Proposition 2.6. Let $h \in C^{2, \mu}(\bar{R})$ be a solution to the problem (4a)- $4 c$ ). If the mapping $q \mapsto h(q, p)$, with any fixed $p \in\left[p_{0}, 0\right]$, is analytic in $\mathbb{R}$, then each streamline including the free surface is an analytic curve. Moreover if $h \in G^{s}(\bar{R})$ then the stream function $\psi$ for (3a)-(3d) lies in $G^{s}(\bar{\Omega})$; in particular $\psi$ is analytic in $\bar{\Omega}$ provided $h$ is analytic in $\bar{R}$.

Proof. The first statement is straightforward. Indeed, Observing (2), each streamline $\psi(x, y)=p$, with fixed $p \in\left[p_{0}, 0\right]$, can be described by the graph of some function $y=$ $\sigma_{p}(x)$. The analyticity of $x \mapsto \sigma_{p}(x)$ follows at once from the analyticity of the mapping $q \mapsto h(q, p)$, due to the partial hodograph change of variables.

As for the second one, we rewrite the hodograph transform as

$$
q=x, \quad p=\tilde{\psi}_{y}
$$

with $\tilde{\psi}(x, y) \stackrel{\text { def }}{=}-\int_{0}^{y} \psi(x, z) d z$. This is just the classic partial Legendre transformation. If $h(q, p) \in G^{s}(\bar{R})$ then $y=y(q, p) \in G^{s}(\bar{R})$. Thus by [2, Theorem 3.1], we have $\tilde{\psi} \in G^{s}(\bar{\Omega})$ and thus $\psi \in G^{s}(\bar{\Omega})$ since $G^{s}(\bar{\Omega})$ is closed under differentiation. 


\subsection{Notations and some useful inequalities}

We list some notations and useful inequalities which will be used throughout the paper. Let $k \in \mathbb{N}$ and $\mu \in(0,1)$, and let $\left(C^{k, \mu}(\bar{R}) ;\|\cdot\|_{k, \mu ; \bar{R}}\right)$ be the standard Hölder space equipped with the norm

$$
\|w\|_{k, \mu ; \bar{R}}=\sum_{|\alpha|=0}^{k} \sup _{\bar{R}}\left|\partial^{\alpha} w(q, p)\right|+\sup _{|\alpha|=k(q, p) \neq(\tilde{q}, \tilde{p})} \sup _{\substack{\bar{R} \\ \mid}} \frac{\left|\partial^{\alpha} w(q, p)-\partial^{\alpha} w(\tilde{q}, \tilde{p})\right|}{|(q, p)-(\tilde{q}, \tilde{p})|^{\mu}} .
$$

To simplify the notation we will use the notation $\|\cdot\|_{k, \mu}$ instead of $\|\cdot\|_{k, \mu ; \bar{R}}$ if no confusion occurs. For the case when $\mu=0$, we naturally define

$$
\|w\|_{k}=\|w\|_{k, 0}=\sum_{|\alpha|=0}^{k} \sup _{\bar{R}}\left|\partial^{\alpha} w\right|
$$

For $\mu \in(0,1)$, direct verification shows that

$$
\|u w\|_{0, \mu} \leq\|u\|_{0, \mu}\|w\|_{0, \mu}, \quad\|u w\|_{1, \mu} \leq 2\|u\|_{1, \mu}\|w\|_{1, \mu} .
$$

For a multi-index $\alpha=\left(\alpha_{1}, \alpha_{2}\right) \in \mathbb{N}^{2}$, we denote $\partial^{\alpha}=\partial_{q}^{\alpha_{1}} \partial_{p}^{\alpha_{2}}, \alpha !=\alpha_{1} ! \alpha_{2}$ ! and denote the length of $\alpha$ by $|\alpha|=\alpha_{1}+\alpha_{2}$. Moreover for two multi-indices $\alpha$ and $\beta=\left(\beta_{1}, \beta_{2}\right) \in \mathbb{N}^{2}$, by $\beta \leq \alpha$ we mean $\beta_{i} \leq \alpha_{i}$ for each $1 \leq i \leq 2$. Let $\left(\begin{array}{l}\alpha \\ \beta\end{array}\right)$ be the binomial coefficient, i.e.,

$$
\left(\begin{array}{l}
\alpha \\
\beta
\end{array}\right)=\frac{\alpha !}{\beta !(\alpha-\beta) !}=\frac{\alpha_{1} ! \alpha_{2} !}{\beta_{1} !\left(\alpha_{1}-\beta_{1}\right) ! \beta_{2} !\left(\alpha_{2}-\beta_{2}\right) !}
$$

In the sequel, we use the convention that $m !=1$ if $m \leq 0$.

\section{Analyticity of streamlines}

We prove in this section the analyticity of streamlines, including the free surface $y=\eta(x)$. In view of Proposition 2.6, it suffices to show the following conclusion that the map $q \mapsto h(q, p)$ is analytic for all $p \in\left[p_{0}, 0\right]$.

Proposition 3.1. Let $\gamma \in C^{0, \mu}\left(\left[p_{0}, 0\right]\right)$ with $p_{0}<0$ and $0<\mu<1$ given, and $h \in C^{2, \mu}(\bar{R})$ be a solution of the governing equations (4a)-(4c). Then there exists a constant $L \geq 1$, such that for all $m \in \mathbb{N}$ with $m \geq 2$, one has the following estimate

$$
\left(E_{m}\right): \quad\left\|\partial_{q}^{m} h\right\|_{2, \mu} \leq L^{m-1}(m-2) !
$$

Thus the map $q \mapsto h(q, p)$ is analytic for all $p \in\left[p_{0}, 0\right]$.

Remark 3.2. As to be seen in the proof below, the constant $L$ depends on $\mu, \inf _{\bar{R}} h_{p}$, $\|h\|_{2, \mu},\|\gamma\|_{0, \mu}$ and the number $\delta$ given in (5), but independent of the order $m$ of derivative. 
Remark 3.3. Starting from the $C^{2, \mu}$-regularity solution $h$ of the governing equations (4a)-(4c), we use the Schauder estimate ( cf. [5, Theorem 6.30]) for $\partial_{q} h$ which satisfies a nonlinear elliptic equation of the same type as (4a)-(4c), to conclude that $\partial_{q} h \in C^{2, \mu}(\bar{R})$. Repeating the procedure, we can derive by standard iteration that $\partial_{q}^{k} h \in C^{2, \mu}(\bar{R})$ for any $k \in \mathbb{N}$; see for instance [4, 10].

To confirm the last statement in the above Proposition 3.1 , we choose $C$ in such a way that

$$
C=\max \left\{L,\|h\|_{1, \mu}\right\}
$$

which, along with the estimate $\left(E_{m}\right)$ with $m \geq 2$ in Proposition 3.1, yields

$$
\forall m \in \mathbb{N}, \quad \max _{(q, p) \in \bar{R}}\left|\partial_{q}^{m} h(q, p)\right| \leq C^{m+1} m ! .
$$

In particular, for any $p \in\left[p_{0}, 0\right], \max _{q \in \mathbb{R}}\left|\partial_{q}^{m} h(q, p)\right| \leq C^{m+1} m$ !. This gives the real analyticity of the map $q \mapsto h(q, p), p \in\left[p_{0}, 0\right]$.

Before proving the above proposition, we first give the following technical lemma, and present its proof at the end of this section.

Lemma 3.4. Let $\ell=1$ or 2 be given, and let $\|\cdot\|$ stand for some Hölder norm $\|\cdot\|_{0, \mu}$ or $\|\cdot\|_{1, \mu}$. Suppose that $k_{0}$ is an integer with $k_{0} \geq \ell+1$, and $\partial_{q}^{k} u_{j} \in C^{0, \mu}(\bar{R})$ for all $k \leq k_{0}$, $j=1,2,3$. If there exists a constant $H \geq 1$ such that

$$
\forall \ell+1 \leq k \leq k_{0}, \quad\left\|\partial_{q}^{k} u_{j}\right\| \leq H^{k-\ell}(k-\ell-1) !, \quad j=1,2,3,
$$

then we can find a constant $C_{*}$ depending only on $\ell$ such that

$$
\forall \ell+1 \leq k \leq k_{0}, \quad\left\|\partial_{q}^{k}\left(u_{1} u_{2} u_{3}\right)\right\| \leq C_{*}\left(\sum_{j=1}^{3}\left\|u_{j}\right\|_{\ell+1, \mu}+1\right)^{6} H^{k-\ell}(k-\ell-1) ! .
$$

We now prove Proposition 3.1.

Proof of Proposition 3.1. In view of Remark 3.3 we may assume that $\partial_{q}^{k} h \in C^{2, \mu}(\bar{R})$ for any $k \in \mathbb{N}$. Now we prove the validity of $\left(E_{m}\right)$ by using induction on $m$. For $m=2,\left(E_{m}\right)$ obviously holds if we choose

$$
L \geq\left\|\partial_{q}^{2} h\right\|_{2, \mu}+1
$$

Now let $m \geq 3$ and assume that $\left(E_{j}\right)$ holds for all $j \in \mathbb{N}$ with $2 \leq j \leq m-1$, that is,

$$
\left\|\partial_{q}^{j} h\right\|_{2, \mu} \leq L^{j-1}(j-2) !, \quad 2 \leq j \leq m-1 .
$$

Then we show the validity of $\left(E_{m}\right)$. For this purpose, taking the derivative with respect to $q$ up to order $m$ on both sides of equations (4a)-(4c), and then applying Leibniz formula, we have

$$
\begin{cases}A(h)\left[\partial_{q}^{m} h\right]=f_{1}+f_{2} & \text { in } R, \\ B(h)\left[\partial_{q}^{m} h\right]=\varphi_{1}+\varphi_{2} & \text { on } p=0, \\ \partial_{q}^{m} h=0 & \text { on } p=p_{0},\end{cases}
$$


where the operators $A(h)$ and $B(h)$ are defined by

$$
A(h)[\phi]=\left(1+h_{q}^{2}\right) \phi_{p p}-2 h_{q} h_{p} \phi_{q p}+h_{p}^{2} \phi_{q q}, \quad B(h)[\phi]=h_{q} \phi_{q}+(2 g h-Q) h_{p} \phi_{p}+2 g h_{p}^{2} \phi,
$$

and the right-hand side

$$
\begin{aligned}
f_{1} & =\sum_{n=1}^{m}\left(\begin{array}{c}
m \\
n
\end{array}\right)\left[-\left(\partial_{q}^{n} h_{q}^{2}\right)\left(\partial_{q}^{m-n} h_{p p}\right)+2\left(\partial_{q}^{n}\left(h_{p} h_{q}\right)\right)\left(\partial_{q}^{m-n} h_{p q}\right)-\left(\partial_{q}^{n} h_{p}^{2}\right)\left(\partial_{q}^{m-n} h_{q q}\right)\right] \\
f_{2} & =-\gamma(p)\left(\partial_{q}^{m} h_{p}^{3}\right) \\
\varphi_{1} & =-\frac{1}{2} \sum_{n=1}^{m-1}\left(\begin{array}{c}
m \\
n
\end{array}\right)\left(\partial_{q}^{n} h_{q}\right)\left(\partial_{q}^{m-n} h_{q}\right)-\frac{1}{2}(2 g h-Q) \sum_{n=1}^{m-1}\left(\begin{array}{c}
m \\
n
\end{array}\right)\left(\partial_{q}^{n} h_{p}\right)\left(\partial_{q}^{m-n} h_{p}\right), \\
\varphi_{2} & =-g \sum_{1 \leq n \leq m-1}\left(\begin{array}{c}
m \\
n
\end{array}\right)\left(\partial_{q}^{n} h\right)\left(\partial_{q}^{m-n} h_{p}^{2}\right) .
\end{aligned}
$$

The operator $A(h)$ is uniformly elliptic since its coefficients satisfy

$$
\left(1+h_{q}^{2}\right) h_{p}^{2}-h_{q}^{2} h_{p}^{2}=h_{p}^{2} \geq \inf _{\bar{R}} h_{p}^{2}>0
$$

due to (5). Also the boundary operator $B(h)$ is uniformly oblique in the sense that it is bounded away from being tangential; the coefficient $(2 g h-Q) h_{p}$ of $\phi_{p}$ is nonzero and satisfies

$$
(2 g h-Q) h_{p}=\frac{1+h_{q}^{2}}{h_{p}} \geq \frac{1}{\sup _{\bar{R}} h_{p}} \geq \delta
$$

in view of the boundary condition (4b) and (15). Since $h \in C^{2, \mu}(\bar{R})$ the coefficients of the operators $A(h)$ and $B(h)$ are in $C^{1, \mu}(\bar{R})$. Moreover, by virtue of the induction assumption (9), one has $\partial_{q}^{i} \partial_{p}^{j} h \in C^{0, \mu}(\bar{R})$ for all multi-index $(i, j)$ with $i+j \leq m+1$ and $j \leq 2$, and similarly $\partial_{q}^{i} \partial_{p}^{j} h \in C^{1, \mu}(\bar{R})$ for all multi-index $(i, j)$ with $i+j \leq m$ and $j \leq 1$. As a result, the right-hand side $f_{i} \in C^{0, \mu}(\bar{R})$ and $\varphi_{i} \in C^{1, \mu}(\bar{R}), i=1,2$, since by (6) the product of two functions in $C^{k, \mu}(\bar{R})$ is still in $C^{k, \mu}(\bar{R})$ with $k=0,1$. Thus, using the standard Schauder estimate (see for instance [5, Theorem 6.30]) we have,

$$
\left\|\partial_{q}^{m} h\right\|_{2, \mu} \leq \mathcal{C}\left(\left\|\partial_{q}^{m} h\right\|_{0}+\sum_{i=1}^{2}\left\|f_{i}\right\|_{0, \mu}+\sum_{i=1}^{2}\left\|\varphi_{i}\right\|_{1, \mu}\right)
$$

where $\mathcal{C}$ is a constant depending only on $\mu, \delta, \inf _{\bar{R}} h_{p}$ and $\|h\|_{2, \mu}$. To show $\left(E_{m}\right)$ is valid, we estimate the terms on the right-hand side of (15) through the following steps.

To simplify the notations, we will use $C_{j}, j \geq 1$, to denote suitable harmless constants larger than 1. By harmless constants we mean these are independent of $m$.

Step 1) We claim that there exists $C_{1}>0$ such that, with $m \geq 3$,

$$
\left\|\partial_{q}^{m} h\right\|_{0} \leq C_{1} L^{m-2}(m-2) !
$$


Indeed, when $m=3$ the above estimate obviously holds if we choose $C_{1}=\|h\|_{3, \mu}+1$; when $m \geq 4$ it follows from the induction assumption (9) that

$$
\left\|\partial_{q}^{m} h\right\|_{0} \leq\left\|\partial_{q}^{m-2} h\right\|_{2, \mu} \leq L^{m-3}(m-4) ! \leq L^{m-2}(m-2) !
$$

Then (16) follows.

Step 2) Let $f_{1}$ be given in (11). In this step we prove

$$
\left\|f_{1}\right\|_{0, \mu} \leq C_{2} L^{m-2}(m-2) !
$$

Observe that , by ([6]),

$$
\begin{array}{r}
\left\|f_{1}\right\|_{0, \mu} \leq \sum_{n=1}^{m}\left(\begin{array}{c}
m \\
n
\end{array}\right)\left\|\partial_{q}^{n} h_{q}^{2}\right\|_{0, \mu}\left\|\partial_{q}^{m-n} h_{p p}\right\|_{0, \mu}+2 \sum_{n=1}^{m}\left(\begin{array}{c}
m \\
n
\end{array}\right)\left\|\partial_{q}^{n}\left(h_{p} h_{q}\right)\right\|_{0, \mu}\left\|\partial_{q}^{m-n} h_{p q}\right\|_{0, \mu} \\
+\sum_{n=1}^{m}\left(\begin{array}{c}
m \\
n
\end{array}\right)\left\|\partial_{q}^{n} h_{p}^{2}\right\|_{0, \mu}\left\|\partial_{q}^{m-n} h_{q q}\right\|_{0, \mu} .
\end{array}
$$

We now treat the first term on the right-hand side, and write

$$
\begin{aligned}
& \sum_{1 \leq n \leq m}\left(\begin{array}{c}
m \\
n
\end{array}\right)\left\|\partial_{q}^{n} h_{q}^{2}\right\|_{0, \mu}\left\|\partial_{q}^{m-n} h_{p p}\right\|_{0, \mu} \leq \sum_{1 \leq n \leq m}\left(\begin{array}{c}
m \\
n
\end{array}\right)\left\|\partial_{q}^{n} h_{q}^{2}\right\|_{0, \mu}\left\|\partial_{q}^{m-n} h\right\|_{2, \mu} \\
& \leq\left(\sum_{1 \leq n \leq 2}+\sum_{3 \leq n \leq m-2}+\sum_{m-1 \leq n \leq m}\right)\left(\begin{array}{c}
m \\
n
\end{array}\right)\left\|\partial_{q}^{n} h_{q}^{2}\right\|_{0, \mu}\left\|\partial_{q}^{m-n} h\right\|_{2, \mu} .
\end{aligned}
$$

By the induction assumption (9), one has

$$
\forall 3 \leq n \leq m, \quad\left\|\partial_{q}^{n} h_{q}\right\|_{0, \mu} \leq\left\|\partial_{q}^{n-1} h\right\|_{2, \mu} \leq L^{n-2}(n-3) ! .
$$

Thus applying Lemma 3.4, with $\ell=2, k_{0}=m, H=L, u_{1}=u_{2}=h_{q}$ and $u_{3}=1$, yields that

$$
\forall 3 \leq n \leq m, \quad\left\|\partial_{q}^{n} h_{q}^{2}\right\|_{0, \mu} \leq C_{5} L^{n-2}(n-3) !
$$

Moreover, we have

$$
\forall 1 \leq n \leq m-2, \quad\left\|\partial_{q}^{m-n} h\right\|_{2, \mu} \leq L^{m-n-1}(m-n-2) !
$$

due to the induction assumption (9). Then using the above two estimates, straightforward verification shows that

$$
\sum_{n=1}^{2}\left(\begin{array}{c}
m \\
n
\end{array}\right)\left\|\partial_{q}^{n} h_{q}^{2}\right\|_{0, \mu}\left\|\partial_{q}^{m-n} h\right\|_{2, \mu}+\sum_{n=m-1}^{m}\left(\begin{array}{c}
m \\
n
\end{array}\right)\left\|\partial_{q}^{n} h_{q}^{2}\right\|_{0, \mu}\left\|\partial_{q}^{m-n} h\right\|_{2, \mu} \leq C_{6} L^{m-2}(m-3) !
$$

if we choose

$$
C_{6} \geq\left(\|h\|_{3, \mu}+1\right)\left(30\|h\|_{3, \mu}+4 C_{5}+6\right)
$$


Next for the case when $3 \leq n \leq m-2$, which appears only when $m \geq 5$, combination of the estimates (20) and (21) gives

$$
\begin{aligned}
\sum_{n=3}^{m-2}\left(\begin{array}{c}
m \\
n
\end{array}\right)\left\|\partial_{q}^{n} h_{q}^{2}\right\|_{0, \mu}\left\|\partial_{q}^{m-n} h\right\|_{2, \mu} & \leq C_{5} \sum_{3 \leq n \leq m-2} \frac{m !}{n !(m-n) !} L^{n-2}(n-3) ! L^{m-n-1}(m-n-2) ! \\
& \leq C_{7} L^{m-3}(m-2) ! \sum_{3 \leq n \leq m-2} \frac{m^{2}}{n^{3}(m-n)^{2}} \\
& \leq C_{8} L^{m-3}(m-2) !
\end{aligned}
$$

This along with (22) shows, in view of (19),

$$
\sum_{1 \leq n \leq m}\left(\begin{array}{c}
m \\
n
\end{array}\right)\left\|\partial_{q}^{n} h_{q}^{2}\right\|_{0, \mu}\left\|\partial_{q}^{m-n} h_{p p}\right\|_{0, \mu} \leq\left(C_{6}+C_{8}\right) L^{m-2}(m-2) !
$$

Similarly, we can find a constant $C_{9}$ such that

$2 \sum_{n=1}^{m}\left(\begin{array}{c}m \\ n\end{array}\right)\left\|\partial_{q}^{n}\left(h_{p} h_{q}\right)\right\|_{0, \mu}\left\|\partial_{q}^{m-n} h_{p q}\right\|_{0, \mu}+\sum_{n=1}^{m}\left(\begin{array}{c}m \\ n\end{array}\right)\left\|\partial_{q}^{n} h_{p}^{2}\right\|_{0, \mu}\left\|\partial_{q}^{m-n} h_{q q}\right\|_{0, \mu} \leq C_{9} L^{m-2}(m-2) !$.

Inserting the above two estimates into (18), we get the desired estimate (17) by choosing $C_{2}=C_{6}+C_{8}+C_{9}$.

Step 3) Let $f_{2}$ be given in (12). We now prove

$$
\left\|f_{2}\right\|_{0, \mu} \leq C_{3} L^{m-2}(m-2) !
$$

In fact, using (6) we have

$$
\left\|f_{2}\right\|_{0, \mu} \leq\|\gamma\|_{0, \mu}\left\|\partial_{q}^{m} h_{p}^{3}\right\|_{0, \mu} .
$$

By the induction assumption (9), one has

$$
\forall 3 \leq j \leq m, \quad\left\|\partial_{q}^{j} h_{p}\right\|_{0, \mu} \leq\left\|\partial_{q}^{j-1} h\right\|_{2, \mu} \leq L^{j-2}(j-3) !
$$

Then using Lemma 3.4, with $\ell=2, k_{0}=m, H=L, u_{1}=u_{2}=u_{3}=h_{p}$, we conclude

$$
\left\|\partial_{q}^{m} h_{p}^{3}\right\|_{0, \mu} \leq C_{10} L^{m-2}(m-3) !
$$

Choosing $C_{3}=C_{10}\|\gamma\|_{0, \mu}+1$, we obtain (23) in view of (24).

Step 4) Finally we prove, with $\varphi_{1}$ and $\varphi_{2}$ given in (13) and (14),

$$
\sum_{i=1}^{2}\left\|\varphi_{i}\right\|_{1, \mu} \leq C_{4} L^{m-2}(m-2) !
$$

First for $\left\|\varphi_{1}\right\|_{1, \mu}$, we have

$$
\left\|\varphi_{1}\right\|_{1, \mu} \leq C_{11} L^{m-2}(m-2) !
$$


The proof is quite similar as that of (17) for $\left\|f_{1}\right\|_{0, \mu}$, and is in fact simpler since we do not need to use Lemma 3.4, so we omit the details. Next for $\left\|\varphi_{2}\right\|_{1, \mu}$, we write, by (하),

$$
\begin{aligned}
\left\|\varphi_{2}\right\|_{1, \mu} & \leq 2 g \sum_{1 \leq n \leq m-1}\left(\begin{array}{c}
m \\
n
\end{array}\right)\left\|\partial_{q}^{n} h\right\|_{1, \mu}\left\|\partial_{q}^{m-n} h_{p}^{2}\right\|_{1, \mu} \\
& \leq 2 g\left(\sum_{1 \leq n \leq 2}+\sum_{3 \leq n \leq m-2}+\sum_{n=m-1}\right)\left(\begin{array}{c}
m \\
n
\end{array}\right)\left\|\partial_{q}^{n} h\right\|_{1, \mu}\left\|\partial_{q}^{m-n} h_{p}^{2}\right\|_{1, \mu} .
\end{aligned}
$$

By the induction assumption (9), one has

$$
\forall 3 \leq n \leq m, \quad\left\|\partial_{q}^{n} h\right\|_{1, \mu} \leq\left\|\partial_{q}^{n-1} h\right\|_{2, \mu} \leq L^{n-2}(n-3) !,
$$

and for $1 \leq n \leq m-2$,

$$
\forall 2 \leq j \leq m-n, \quad\left\|\partial_{q}^{j} h_{p}\right\|_{1, \mu} \leq\left\|\partial_{q}^{j} h\right\|_{2, \mu} \leq L^{j-1}(j-2) ! .
$$

This last estimate allows us to use Lemma 3.4, with $\ell=1, k_{0}=m-n$ with $1 \leq n \leq m-2$ , $H=L, u_{1}=u_{2}=h_{p}$ and $u_{3}=1$, to conclude,

$$
\forall 1 \leq n \leq m-2, \quad\left\|\partial_{q}^{m-n} h_{p}^{2}\right\|_{1, \mu} \leq C_{12} L^{m-n-1}(m-n-2) ! .
$$

In virtue of (27) and (28), direct verification shows

$$
2 g\left(\sum_{1 \leq n \leq 2}+\sum_{n=m-1}\right)\left(\begin{array}{c}
m \\
n
\end{array}\right)\left\|\partial_{q}^{n} h\right\|_{1, \mu}\left\|\partial_{q}^{m-n} h_{p}^{2}\right\|_{1, \mu} \leq C_{13} L^{m-2}(m-2) ! .
$$

Next for the case when $3 \leq n \leq m-2$, which appears only when $m \geq 5$, we use again (27) and (28) to compute

$$
\begin{aligned}
& 2 g \sum_{3 \leq n \leq m-2}\left(\begin{array}{c}
m \\
n
\end{array}\right)\left\|\partial_{q}^{n} h\right\|_{1, \mu}\left\|\partial_{q}^{m-n} h_{p}^{2}\right\|_{1, \mu} \\
\leq & 2 g C_{12} \sum_{3 \leq n \leq m-2} \frac{m !}{n !(m-n) !} L^{n-2}(n-3) ! L^{m-n-1}(m-n-2) ! \\
\leq & C_{14} L^{m-3}(m-2) ! \sum_{3 \leq n \leq m-2} \frac{m^{2}}{n^{3}(m-n)^{2}} \\
\leq & C_{15} L^{m-3}(m-2) ! .
\end{aligned}
$$

Inserting (29) and the above estimate into (26), we obtain

$$
\left\|\varphi_{2}\right\|_{1, \mu} \leq\left(C_{13}+C_{15}\right) L^{m-2}(m-2) !
$$

Thus the desired estimate (25) follows by choosing $C_{4}=C_{11}+C_{13}+C_{15}$.

Now we come back to the proof of Proposition 3.1. Choose $L$ in such a way that

$$
L \geq \mathcal{C}\left(C_{1}+C_{2}+C_{3}+C_{4}\right)+\left\|\partial_{q}^{2} h\right\|_{2, \mu}+1
$$


with $\mathcal{C}, C_{1}, \cdots, C_{4}$ the constants given in (15), (16), (17), (23) and (25). Then combining (15), (16), (17), (23) and (25), we have,

$$
\left\|\partial_{q}^{m} h\right\|_{2, \mu} \leq \mathcal{C}\left(C_{1}+C_{2}+C_{3}+C_{4}\right) L^{m-2}(m-2) ! \leq L^{m-1}(m-2) ! .
$$

The validity of $\left(E_{m}\right)$ follows. Thus the proof of Proposition 3.1 is complete.

The rest of this section is occupied by

Proof of Lemma 3.4. In what follows we always assume $\ell+1 \leq k \leq k_{0}$. To simplify the notation, we use $b_{i}, i \geq 1$, to denote suitable constants larger than 1 , depending only on $\ell$.

Firstly let $u_{3} \equiv 1$. By Leibniz formula we have

$$
\partial_{q}^{k}\left(u_{1} u_{2}\right)=\sum_{0 \leq j \leq k}\left(\begin{array}{l}
k \\
j
\end{array}\right)\left(\partial_{q}^{j} u_{1}\right)\left(\partial_{q}^{k-j} u_{2}\right) .
$$

Note that $\|\cdot\|$ stands for the Hölder norm $\|\cdot\|_{0, \mu}$ or $\|\cdot\|_{1, \mu}$. Then from (6) it follows that

$$
\begin{aligned}
\left\|\partial_{q}^{k}\left(u_{1} u_{2}\right)\right\| & \leq 2 \sum_{0 \leq j \leq k} \frac{k !}{j !(k-j) !}\left\|\partial_{q}^{j} u_{1}\right\|\left\|\partial_{q}^{k-j} u_{2}\right\| \\
& \leq S_{1}+S_{2}+S_{3}
\end{aligned}
$$

with

$$
\begin{aligned}
S_{1} & =2 \sum_{0 \leq j \leq \ell} \frac{k !}{j !(k-j) !}\left\|\partial_{q}^{j} u_{1}\right\|\left\|\partial_{q}^{k-j} u_{2}\right\|, \\
S_{2} & =2 \sum_{\ell+1 \leq j \leq k-\ell-1} \frac{k !}{j !(k-j) !}\left\|\partial_{q}^{j} u_{1}\right\|\left\|\partial_{q}^{k-j} u_{2}\right\|, \\
S_{3} & =2 \sum_{k-\ell \leq j \leq k} \frac{k !}{j !(k-j) !}\left\|\partial_{q}^{j} u_{1}\right\|\left\|\partial_{q}^{k-j} u_{2}\right\| .
\end{aligned}
$$

Using the assumption (8), direct computation shows that there exists a constant $b_{1}>1$, depending only on $\ell$, such that

$$
S_{1}+S_{3} \leq b_{1}\left(\left\|u_{1}\right\|_{\ell+1, \mu}+\left\|u_{2}\right\|_{\ell+1, \mu}+1\right)^{2} H^{k-\ell}(k-\ell-1) ! .
$$

For $S_{2}$, which appears only when $k \geq 2 \ell+2$, we have

$$
\begin{aligned}
S_{2} & \leq 2 \sum_{\ell+1 \leq j \leq k-\ell-1} \frac{k !}{j !(k-j) !} H^{j-\ell}(j-\ell-1) ! H^{k-j-\ell}(k-j-\ell-1) ! \\
& \leq b_{2} \sum_{\ell+1 \leq j \leq k-\ell-1} \frac{k !}{j^{\ell+1}(k-j)^{\ell+1}} H^{k-2 \ell} \\
& \leq b_{3} H^{k-2 \ell}(k-\ell-1) ! \sum_{\ell+1 \leq j \leq k-\ell-1} \frac{k^{\ell+1}}{j^{\ell+1}(k-j)^{\ell+1}} \\
& \leq b_{4} H^{k-\ell}(k-\ell-1) ! .
\end{aligned}
$$


In view of the estimates for $S_{1}, S_{2}$ and $S_{3}$, we conclude

$$
\left\|\partial_{q}^{k}\left(u_{1} u_{2}\right)\right\| \leq b_{5}\left(\left\|u_{1}\right\|_{\ell+1, \mu}+\left\|u_{2}\right\|_{\ell+1, \mu}+1\right)^{2} H^{k-\ell}(k-\ell-1) !
$$

by choosing $b_{5}=b_{1}+b_{4}$.

Now we consider the case when $u_{3} \not \equiv 1$. We have shown above that

$$
\forall \ell+1 \leq k \leq k_{0}, \quad\left\|\partial_{q}^{k}\left(u_{1} u_{2}\right)\right\| \leq b_{5}\left(\left\|u_{1}\right\|_{\ell+1, \mu}+\left\|u_{2}\right\|_{\ell+1, \mu}+1\right)^{2} H^{k-\ell}(k-\ell-1) !,
$$

provided $u_{1}$ and $u_{2}$ satisfy (8). This allows us to use the same argument as above to the two functions

$$
b_{5}^{-1}\left(\left\|u_{1}\right\|_{\ell+1, \mu}+\left\|u_{2}\right\|_{\ell+1, \mu}+1\right)^{-2} u_{1} u_{2} \text { and } u_{3} ;
$$

this gives, for any $\ell+1 \leq k \leq k_{0}$,

$$
\begin{aligned}
\left\|\partial_{q}^{k}\left(u_{1} u_{2} u_{3}\right)\right\| & \leq b_{5}^{2}\left(\left\|u_{1}\right\|_{\ell+1, \mu}+\left\|u_{2}\right\|_{\ell+1, \mu}+1\right)^{2}\left(\left\|u_{1} u_{2}\right\|_{\ell+1, \mu}+\left\|u_{3}\right\|_{\ell+1, \mu}+1\right)^{2} H^{k-\ell}(k-\ell-1) ! \\
& \leq b_{6}\left(\left\|u_{1}\right\|_{\ell+1, \mu}+\left\|u_{2}\right\|_{\ell+1, \mu}+\left\|u_{3}\right\|_{\ell+1, \mu}+1\right)^{6} H^{k-\ell}(k-\ell-1) ! .
\end{aligned}
$$

The conclusion follows by choosing $C_{*}=b_{6}$. Then the proof of Lemma 3.4 is complete.

\section{Gevrey regularity of stream function}

Let $G^{s}\left(\left[p_{0}, 0\right]\right), s \geq 1$, be the Gevrey class; see Definition 2.1 of Gevrey function. In this section we assume $\gamma \in G^{s}\left(\left[p_{0}, 0\right]\right)$. Then by the alternative characterization of Gevrey function, for any $p \in\left[p_{0}, 0\right]$ we can find a neighborhood $U_{p}$ of $p$ and a constant $M_{p}$ such that

$$
\forall k \in \mathbb{N}, \quad \sup _{t \in U_{p} \cap\left[p_{0}, 0\right]}\left|\partial_{p}^{k} \gamma(t)\right| \leq M_{p}^{k+1}(k !)^{s} .
$$

Note $\left[p_{0}, 0\right]$ is compact in $\mathbb{R}$; this allows us to find a constant $M$ such that

$$
\forall k \in \mathbb{N}, \quad \sup _{p \in\left[p_{0}, 0\right]}\left|\partial_{p}^{k} \gamma(p)\right| \leq M^{k+1}(k !)^{s} .
$$

We prove now the Gevrey regularity of stream function, i.e., Theorem 2.4. In view of Proposition 2.6, it suffices to show the following result for the height function $h(q, p)$.

Proposition 4.1. Let $\gamma \in G^{s}\left(\left[p_{0}, 0\right]\right)$ with $s \geq 1$, and let $h \in C^{2, \mu}(\bar{R})$ be a solution to (4a)-(4c). Then there exist two constants $L_{1}, L_{2}$ with $L_{2} \geq L_{1} \geq 1$, such that for any $m \geq 2$ we have the following estimate

$$
\left(F_{m}\right): \quad \forall \alpha=\left(\alpha_{1}, \alpha_{2}\right) \in \mathbb{N}^{2}, \quad|\alpha|=m, \quad\left\|\partial^{\alpha} h\right\|_{2} \leq L_{1}^{\alpha_{1}-1} L_{2}^{\alpha_{2}}[(|\alpha|-2) !]^{s} .
$$

Recall $\|\cdot\|_{2}$ stands for the Hölder norm $\|\cdot\|_{C^{2,0}(\bar{R})}$. Thus $h \in G^{s}(\bar{R})$; in particular if $s=1$ then $h$ is analytic in $\bar{R}$. 
Remark 4.2. As to be seen in the proof, the constants $L_{1}, L_{2}$ depend on the constant $L$ given in Proposition 3.1 and the constant $M$ in (30), but independent of the order $m$ of derivative.

Remark 4.3. Note $\gamma \in G^{s}\left(\left[p_{0}, 0\right]\right) \subset C^{\infty}\left(\left[p_{0}, 0\right]\right)$. By Remark 3.3 we see $\partial_{q} h \in C^{2, \mu}(\bar{R})$. Then differentiating the equation (4a) with respect to $p$, we can obtain $h \in C^{3, \mu}(\bar{R})$; see [4] for details. Repeating this procedure gives $h \in C^{k, \mu}(\bar{R})$ for any $k \in \mathbb{N}$, since $\gamma \in C^{\infty}\left(\left[p_{0}, 0\right]\right)$.

To confirm the last statement in the above Proposition 4.1, we choose $C$ in such a way that

$$
C=\max \left\{L_{1}, L_{2},\|h\|_{1, \mu}\right\},
$$

which, along with the estimate $\left(F_{m}\right)$ with $m \geq 2$ in Proposition 4.1, yields

$$
\forall \alpha \in \mathbb{N}^{2}, \quad \max _{(q, p) \in \bar{R}}\left|\partial^{\alpha} h(q, p)\right| \leq C^{|\alpha|+1}(|\alpha| !)^{s} .
$$

This gives $h \in G^{s}(\bar{R})$.

In order to prove the above proposition, we need the following technical lemma.

Lemma 4.4. Let $s \geq 1$, and $H_{1}$ and $H_{2}$ be two constants with $H_{2} \geq H_{1} \geq 1$. Suppose that $\alpha_{0}$ is a given multi-index with $\left|\alpha_{0}\right| \geq 3$, and $u, v, w \in C^{\left|\alpha_{0}\right|, \mu}(\bar{R})$. For $j=0,1,2$, denote

$\mathcal{A}_{j}=\left\{f \in C^{\left|\alpha_{0}\right|, \mu}(\bar{R})\left|\forall \alpha=\left(\alpha_{1}, \alpha_{2}\right) \leq \alpha_{0},\right| \alpha \mid \geq j+1,\left\|\partial^{\alpha} f\right\|_{0} \leq H_{1}^{\alpha_{1}-j} H_{2}^{\alpha_{2}}[(|\alpha|-j-1) !]^{s}\right\}$.

Then there exists a constant $c_{*}$, depending only on the $C^{2,0}$-norms of $u, v$ and $w$, but independent of $\alpha_{0}$, such that

(a) if $u \in \mathcal{A}_{2}$ and $v \in \mathcal{A}_{1}$, then $c_{*}^{-1} u v \in \mathcal{A}_{1}$, that is,

$$
\forall \alpha=\left(\alpha_{1}, \alpha_{2}\right) \leq \alpha_{0}, \quad|\alpha| \geq 2, \quad\left\|\partial^{\alpha}(u v)\right\|_{0} \leq c_{*} H_{1}^{\alpha_{1}-1} H_{2}^{\alpha_{2}}[(|\alpha|-2) !]^{s} ;
$$

if additionally $w \in \mathcal{A}_{2}$ then $c_{*}^{-1} u v w \in \mathcal{A}_{1}$;

(b) if $u \in \mathcal{A}_{2}$ and $v, w \in \mathcal{A}_{1}$, then $c_{*}^{-1} u v w \in \mathcal{A}_{0}$;

(c) if $u, v \in \mathcal{A}_{2}$ and $w \in \mathcal{A}_{0}$, then $c_{*}^{-1} u v w \in \mathcal{A}_{0}$;

(d) if $u, v, w \in \mathcal{A}_{2}$, then $c_{*}^{-1} H_{1} u v w \in \mathcal{A}_{1}$, that is,

$$
\forall \alpha=\left(\alpha_{1}, \alpha_{2}\right) \leq \alpha_{0}, \quad|\alpha| \geq 2, \quad\left\|\partial^{\alpha}(u v w)\right\|_{0} \leq c_{*} H_{1}^{\alpha_{1}-2} H_{2}^{\alpha_{2}}[(|\alpha|-2) !]^{s} .
$$

The proof of the above lemma is postponed to the end of this section. Now we prove our main result. 
Proof of Proposition 4.1. In view of Remark 4.3 we may assume that $h \in C^{k, \mu}(\bar{R})$ for any $k \in \mathbb{N}$. We now use induction on $m$ to prove the estimate $\left(F_{m}\right)$. First for $m=2,\left(F_{m}\right)$ obviously holds by choosing $L_{1}, L_{2}$ in such a way that

$$
L_{2} \geq L_{1} \geq\|h\|_{4}+1
$$

Next let $m \geq 3$ and assume that $\left(F_{j}\right)$ holds for any $j$ with $2 \leq j \leq m-1$, that is,

$$
\forall \beta=\left(\beta_{1}, \beta_{2}\right), 2 \leq \beta_{1}+\beta_{2} \leq m-1, \quad\left\|\partial^{\beta} h\right\|_{2} \leq L_{1}^{\beta_{1}-1} L_{1}^{\beta_{2}}[(|\beta|-2) !]^{s} .
$$

We have to prove the validity of $\left(F_{m}\right)$. This is equivalent to show the following estimate

$$
\left(F_{m, n}\right): \quad\left\|\partial_{q}^{m-n} \partial_{p}^{n} h\right\|_{2} \leq L_{1}^{m-n-1} L_{2}^{n}[(m-2) !]^{s}
$$

holds for all $n$ with $0 \leq n \leq m$.

In what follows we use induction on $n$ to show (33) with fixed $m \geq 3$. Firstly note that $s \geq 1$, and thus from Proposition 3.1 we see that $\left(F_{m, 0}\right)$ holds if we choose

$$
L_{1} \geq L
$$

Next let $1 \leq n \leq m$ and assume that $\left(F_{m, i}\right)$ holds for all $i$ with $0 \leq i \leq n-1$, that is,

$$
\forall 0 \leq i \leq n-1, \quad\left\|\partial_{q}^{m-i} \partial_{p}^{i} h\right\|_{2} \leq L_{1}^{m-i-1} L_{2}^{i}[(m-2) !]^{s} .
$$

We have to show $\left(F_{m, n}\right)$ holds as well, i.e., to prove that

$$
\left\|\partial_{q}^{m-n} \partial_{p}^{n} h\right\|_{2} \leq L_{1}^{m-n-1} L_{2}^{n}[(m-2) !]^{s} .
$$

To do so, we firstly compute, with $1 \leq n \leq m$,

$$
\begin{aligned}
\left\|\partial_{q}^{m-n} \partial_{p}^{n} h\right\|_{2} & \leq\left\|\partial_{q}^{m-n} \partial_{p}^{n} h\right\|_{1}+\left\|\partial_{q}^{m-n+2} \partial_{p}^{n} h\right\|_{0}+\left\|\partial_{q}^{m-n+1} \partial_{p}^{n+1} h\right\|_{0}+\left\|\partial_{q}^{m-n} \partial_{p}^{n+2} h\right\|_{0} \\
& \leq\left\|\partial_{q}^{m-n} \partial_{p}^{n-1} h\right\|_{2}+2\left\|\partial_{q}^{m-(n-1)} \partial_{p}^{n-1} h\right\|_{2}+\left\|\partial_{q}^{m-n} \partial_{p}^{n+2} h\right\|_{0} .
\end{aligned}
$$

The induction assumptions (32) and (35) yield

$$
\begin{aligned}
\left\|\partial_{q}^{m-n} \partial_{p}^{n-1} h\right\|_{2}+2\left\|\partial_{q}^{m-(n-1)} \partial_{p}^{n-1} h\right\|_{2} & \leq L_{1}^{m-n-1} L_{2}^{n-1}[(m-3) !]^{s}+2 L_{1}^{m-n} L_{2}^{n-1}[(m-2) !]^{s} \\
& \leq L_{2}^{-1}\left(1+2 L_{1}\right) L_{1}^{m-n-1} L_{2}^{n}[(m-2) !]^{s} \\
& \leq \frac{1}{2} L_{1}^{m-n-1} L_{2}^{n}[(m-2) !]^{s}
\end{aligned}
$$

where in the last inequality we choose

$$
L_{2} \geq 8 L_{1} \geq 8
$$

Accordingly, in order to obtain (

$$
\left\|\partial_{q}^{m-n} \partial_{p}^{n+2} h\right\|_{0} \leq \frac{1}{2} L_{1}^{m-n-1} L_{2}^{n}[(m-2) !]^{s} .
$$


The rest is occupied by the proof of the above estimate.

From now on we fix $m$ and $n$ with $m \geq 3$ and $1 \leq n \leq m$, and denote $\alpha=\left(\alpha_{1}, \alpha_{2}\right)=$ $(m-n, n)$. Applying $\partial^{\alpha}=\partial_{q}^{m-n} \partial_{p}^{n}$ on both sides of the equation (4a) gives

$$
\left(1+h_{q}^{2}\right)\left(\partial^{\alpha} h_{p p}\right)=-\sum_{\beta \leq \alpha, \beta \neq 0}\left(\begin{array}{c}
\alpha \\
\beta
\end{array}\right)\left(\partial^{\beta} h_{q}^{2}\right)\left(\partial^{\alpha-\beta} h_{p p}\right)+2 \partial^{\alpha}\left(h_{p} h_{q} h_{q p}\right)-\partial^{\alpha}\left(h_{p}^{2} h_{q q}\right)-\partial^{\alpha}\left(\gamma h_{p}^{3}\right),
$$

which implies

$$
\begin{aligned}
\left\|\left(1+h_{q}^{2}\right)\left(\partial^{\alpha} h_{p p}\right)\right\|_{0} \leq & \sum_{\substack{\beta \leq \alpha, \beta \neq 0 \\
\beta}}\left(\begin{array}{c}
\alpha \\
\beta
\end{array}\right)\left\|\partial^{\beta} h_{q}^{2}\right\|_{0}\left\|\partial^{\alpha-\beta} h_{p p}\right\|_{0}+2\left\|\partial^{\alpha}\left(h_{p} h_{q} h_{q p}\right)\right\|_{0} \\
& +\left\|\partial^{\alpha}\left(h_{p}^{2} h_{q q}\right)\right\|_{0}+\left\|\partial^{\alpha}\left(\gamma h_{p}^{3}\right)\right\|_{0} .
\end{aligned}
$$

Since

$$
\left\|\partial_{q}^{m-n} \partial_{p}^{n+2} h\right\|_{0}=\left\|\partial^{\alpha} h_{p p}\right\|_{0} \leq\left\|\left(1+h_{q}^{2}\right)\left(\partial^{\alpha} h_{p p}\right)\right\|_{0},
$$

we obtain, with $\alpha=\left(\alpha_{1}, \alpha_{2}\right)=(m-n, n)$,

$$
\begin{aligned}
\left\|\partial_{q}^{m-n} \partial_{p}^{n+2} h\right\|_{0} \leq & \sum_{\beta \leq \alpha, \beta \neq 0}\left(\begin{array}{c}
\alpha \\
\beta
\end{array}\right)\left\|\partial^{\beta} h_{q}^{2}\right\|_{0}\left\|\partial^{\alpha-\beta} h_{p p}\right\|_{0}+2\left\|\partial^{\alpha}\left(h_{p} h_{q} h_{q p}\right)\right\|_{0} \\
& +\left\|\partial^{\alpha}\left(h_{p}^{2} h_{q q}\right)\right\|_{0}+\left\|\partial^{\alpha}\left(\gamma h_{p}^{3}\right)\right\|_{0} .
\end{aligned}
$$

We now treat the terms on the right-hand side through the following lemmas.

To simplify the notations, we will use $c_{j}, j \geq 1$, to denote suitable harmless constants larger than 1. By harmless constants it means that these constants are independent of $m$ and $n$.

Lemma 4.5. For $\alpha=\left(\alpha_{1}, \alpha_{2}\right)=(m-n, n)$ with $1 \leq n \leq m$, we have

$$
\sum_{\beta \leq \alpha, \beta \neq 0}\left(\begin{array}{c}
\alpha \\
\beta
\end{array}\right)\left\|\partial^{\beta} h_{q}^{2}\right\|_{0}\left\|\partial^{\alpha-\beta} h_{p p}\right\|_{0} \leq c_{1} L_{1}^{\alpha_{1}-2} L_{2}^{\alpha_{2}}[(|\alpha|-2) !]^{s} .
$$

Proof of the lemma. We firstly use Lemma 4.4 to treat the term $\left\|\partial^{\beta} h_{q}^{2}\right\|_{0}$ with $3 \leq|\beta| \leq$ $|\alpha|=m$. To do so, write $\beta=\tilde{\beta}+(\beta-\tilde{\beta})$ with $|\tilde{\beta}|=|\beta|-1 \geq 2$. Without loss of generality we may take $\beta-\tilde{\beta}=(0,1)$, and the arguments below also holds when $\beta-\tilde{\beta}=(1,0)$. Thus

$$
\partial^{\beta} h_{q}^{2}=2 \partial^{\tilde{\beta}}\left(h_{q} h_{q p}\right) .
$$

Note that for any $\xi=\left(\xi_{1}, \xi_{2}\right) \leq \tilde{\beta}$ with $|\xi| \geq 3$, we have, using the induction assumption (32),

$$
\left\|\partial^{\xi} h_{q p}\right\|_{0} \leq\left\|\partial^{\xi} h\right\|_{2} \leq L_{1}^{\xi_{1}-1} L_{2}^{\xi_{2}}[(|\xi|-2) !]^{s}
$$

and

$$
\left\|\partial^{\xi} h_{q}\right\|_{0} \leq \begin{cases}\left\|\partial_{q}^{\xi_{1}} \partial_{p}^{\xi_{2}-1} h\right\|_{2} \leq L_{1}^{\xi_{1}-1} L_{2}^{\xi_{2}-1}[(|\xi|-3) !]^{s} \leq L_{1}^{\xi_{1}-2} L_{2}^{\xi_{2}}[(|\xi|-3) !]^{s}, & \xi_{2} \geq 1 \\ \left\|\partial_{q}^{\xi_{1}-1} h\right\|_{2} \leq L_{1}^{\xi_{1}-2}[(|\xi|-3) !]^{s}=L_{1}^{\xi_{1}-2} L_{2}^{\xi_{2}}[(|\xi|-3) !]^{s}, & \xi_{2}=0\end{cases}
$$


where in the case $\xi_{2} \geq 1$ we used $L_{2} \geq L_{1}$. Therefore applying Lemma 4.4t(国), with $H_{1}=L_{1}, H_{2}=L_{2}, u=h_{q}$ and $v=h_{q p}$, gives

$$
\left\|\partial^{\tilde{\beta}}\left(h_{q} h_{q p}\right)\right\|_{0} \leq c_{4} L_{1}^{\tilde{\beta}_{1}-1} L_{2}^{\tilde{\beta}_{2}}[(|\tilde{\beta}|-2) !]^{s}=c_{4} L_{1}^{\beta_{1}-1} L_{2}^{\beta_{2}-1}[(|\beta|-3) !]^{s} \leq c_{4} L_{1}^{\beta_{1}-2} L_{2}^{\beta_{2}}[(|\beta|-3) !]^{s},
$$

the last inequality holding because $L_{2} \geq L_{1}$. This along with the relation (41) yields

$$
\forall \beta, 3 \leq|\beta| \leq|\alpha|, \quad\left\|\partial^{\beta} h_{q}^{2}\right\|_{0} \leq 2 c_{4} L_{1}^{\beta_{1}-2} L_{2}^{\beta_{2}}[(|\beta|-3) !]^{s} .
$$

On the other hand, for the term $\left\|\partial^{\alpha-\beta} h_{p p}\right\|_{0}$, we have, by the induction assumption (32),

$$
\forall \beta \leq \alpha, 1 \leq|\beta| \leq|\alpha|-2, \quad\left\|\partial^{\alpha-\beta} h_{p p}\right\|_{0} \leq\left\|\partial^{\alpha-\beta} h\right\|_{2} \leq L_{1}^{\alpha_{1}-\beta_{1}-1} L_{2}^{\alpha_{2}-\beta_{2}}[(|\alpha|-|\beta|-2) !]^{s} .
$$

Next we write

$$
\begin{aligned}
\sum_{\beta \leq \alpha, \beta \neq 0}\left(\begin{array}{c}
\alpha \\
\beta
\end{array}\right)\left\|\partial^{\beta} h_{q}^{2}\right\|_{0}\left\|\partial^{\alpha-\beta} h_{p p}\right\|_{0} & =\left(\sum_{\substack{\beta \leq \alpha \\
1 \leq|\beta| \leq 2}}+\sum_{\substack{\beta \leq \alpha \\
3 \leq|\beta| \leq|\alpha|-2}}+\sum_{\substack{\beta \leq \alpha \\
|\beta| \geq|\alpha|-1}}\right)\left(\begin{array}{c}
\alpha \\
\beta
\end{array}\right)\left\|\partial^{\beta} h_{q}^{2}\right\|_{0}\left\|\partial^{\alpha-\beta} h_{p p}\right\|_{0} \\
& =J_{1}+J_{2}+J_{3} .
\end{aligned}
$$

By virtue of (43) and (44), direct computation as in (22), shows that

$$
J_{1}+J_{3} \leq c_{5} L_{1}^{\alpha_{1}-2} L_{2}^{\alpha_{2}}[(|\alpha|-2) !]^{s} .
$$

Next for $J_{2}$, which appears only when $|\alpha| \geq 5$, we have by (43) and (44) that

$$
\begin{aligned}
J_{2} & \leq 2 c_{4} \sum_{\substack{\beta \leq \alpha \\
3 \leq|\beta| \leq|\alpha|-2}} \frac{|\alpha| !}{|\beta| !(|\alpha|-|\beta|) !} L_{1}^{\beta_{1}-2} L_{2}^{\beta_{2}}[(|\beta|-3) !]^{s} L_{1}^{\alpha_{1}-\beta_{1}-1} L_{2}^{\alpha_{2}-\beta_{2}}[(|\alpha|-|\beta|-2) !]^{s} \\
& \leq c_{6} \sum_{\substack{\beta \leq \alpha \\
3 \leq|\beta| \leq|\alpha|-2}} \frac{|\alpha| !}{|\beta|^{3}(|\alpha|-|\beta|)^{2}} L_{1}^{\alpha_{1}-3} L_{2}^{\alpha_{2}}[(|\beta|-3) !]^{s-1}[(|\alpha|-|\beta|-2) !]^{s-1} \\
& \leq c_{6} L_{1}^{\alpha_{1}-3} L_{2}^{\alpha_{2}} \sum_{\substack{\beta \leq \alpha \\
3 \leq|\beta| \leq|\alpha|-2}} \frac{|\alpha| !}{|\beta|^{3}(|\alpha|-|\beta|)^{2}}[(|\alpha|-5) !]^{s-1} \\
& \leq c_{6} L_{1}^{\alpha_{1}-3} L_{2}^{\alpha_{2}}[(|\alpha|-2) !]^{s} \sum_{\substack{\beta \leq \alpha \\
3 \leq|\beta| \leq|\alpha|-2}} \frac{|\beta|^{3}(|\alpha|-|\beta|)^{2}}{\mid{ }^{2}} \\
& \leq c_{7} L_{1}^{\alpha_{1}-3} L_{2}^{\alpha_{2}}[(|\alpha|-2) !]^{s},
\end{aligned}
$$

the last inequality holding because

$$
\sum_{\substack{\beta \leq \alpha \\ 3 \leq|\beta| \leq|\alpha|-2}} \frac{|\alpha|^{2}}{|\beta|^{3}(|\alpha|-|\beta|)^{2}} \leq 8 \pi^{2} .
$$

Therefore, choosing $c_{1}=c_{5}+c_{7}$, we can combine the estimates for $J_{1}, J_{2}$ and $J_{3}$ to complete the proof of the lemma. 
Lemma 4.6. For $\alpha=\left(\alpha_{1}, \alpha_{2}\right)=(m-n, n)$ with $1 \leq n \leq m$, we have

$$
2\left\|\partial^{\alpha}\left(h_{p} h_{q} h_{q p}\right)\right\|_{0}+\left\|\partial^{\alpha}\left(h_{p}^{2} h_{q q}\right)\right\|_{0} \leq c_{2} L_{1}^{\alpha_{1}} L_{2}^{\alpha_{2}-1}[(|\alpha|-2) !]^{s} .
$$

Proof of the lemma. Since $n \geq 1$, we can write $\alpha=\tilde{\alpha}+(0,1)$ with $\tilde{\alpha}=\left(\tilde{\alpha}_{1}, \tilde{\alpha}_{2}\right)=(m-$ $n, n-1)$. Thus

$$
\partial^{\alpha}\left(h_{p} h_{q} h_{q p}\right)=\partial^{\tilde{\alpha}}\left(h_{p p} h_{q} h_{q p}+h_{p} h_{q p} h_{q p}+h_{p} h_{q} h_{q p p}\right) .
$$

We next compute the estimate for the term $\partial^{\tilde{\alpha}}\left(h_{p p} h_{q} h_{q p}\right)$. For any $\beta \leq \tilde{\alpha}$ with $|\beta| \geq 3$, we have, as for $\left\|\partial^{\xi} h_{q}\right\|_{0}$ in (42),

$$
\left\|\partial^{\beta} h_{q}\right\|_{0} \leq L_{1}^{\beta_{1}-2} L_{2}^{\beta_{2}}[(|\beta|-3) !]^{s}, \quad\left\|\partial^{\beta} h_{p}\right\|_{0} \leq L_{1}^{\beta_{1}-2} L_{2}^{\beta_{2}}[(|\beta|-3) !]^{s},
$$

and by the induction assumption (32) and (35), in view of $\beta_{2} \leq \tilde{\alpha}_{2}=n-1$,

$$
\begin{aligned}
\left\|\partial^{\beta} h_{p p}\right\|_{0} & \leq\left\|\partial^{\beta} h\right\|_{2} \leq L_{1}^{\beta_{1}-1} L_{2}^{\beta_{2}}[(|\beta|-2) !]^{s}, \\
\left\|\partial^{\beta} h_{q p}\right\|_{0} & \leq\left\|\partial^{\beta} h\right\|_{2} \leq L_{1}^{\beta_{1}-1} L_{2}^{\beta_{2}}[(|\beta|-2) !]^{s}, \\
\left\|\partial^{\beta} h_{q p p}\right\|_{0} & \leq\left\|\partial_{q}^{\beta_{1}+1} \partial_{p}^{\beta_{2}} h\right\|_{2} \leq L_{1}^{\beta_{1}} L_{2}^{\beta_{2}}[(|\beta|-1) !]^{s} .
\end{aligned}
$$

Thus we obtain, using Lemma 4.4.(国) with $u=h_{p}, w=h_{p}$ and $v=h_{q p}$,

$\left\|\partial^{\tilde{\alpha}}\left(h_{p} h_{q} h_{q p}\right)\right\|_{0} \leq c_{8} L_{1}^{\tilde{\alpha}_{1}-1} L_{2}^{\tilde{\alpha}_{2}}[(|\tilde{\alpha}|-2) !]^{s}=c_{8} L_{1}^{\alpha_{1}-1} L_{2}^{\alpha_{2}-1}[(|\alpha|-3) !]^{s} \leq c_{8} L_{1}^{\alpha_{1}} L_{2}^{\alpha_{2}-1}[(|\alpha|-2) !]^{s}$.

Similarly, using Lemma 4.4-(b) with $u=h_{p}, v=w=h_{q p}$, gives

$$
\left\|\partial^{\tilde{\alpha}}\left(h_{p} h_{q p} h_{q p}\right)\right\|_{0} \leq c_{9} L_{1}^{\tilde{\alpha}_{1}} L_{2}^{\tilde{\alpha}_{2}}[(|\tilde{\alpha}|-1) !]^{s}=c_{9} L_{1}^{\alpha_{1}} L_{2}^{\alpha_{2}-1}[(|\alpha|-2) !]^{s}
$$

while using Lemma 4.4. (ㄷ) with $u=h_{p}, v=h_{q}$ and $w=h_{q p p}$ gives

$$
\left\|\partial^{\tilde{\alpha}}\left(h_{p} h_{p} h_{q p p}\right)\right\|_{0} \leq c_{10} L_{1}^{\tilde{\alpha}_{1}} L_{2}^{\tilde{\alpha}_{2}}[(|\tilde{\alpha}|-1) !]^{s}=c_{10} L_{1}^{\alpha_{1}} L_{2}^{\alpha_{2}-1}[(|\alpha|-2) !]^{s} .
$$

Combining the above inequalities, we have, in view of (45),

$$
2\left\|\partial^{\alpha}\left(h_{p} h_{q} h_{q p}\right)\right\|_{0} \leq 2\left(c_{8}+c_{9}+c_{10}\right) L_{1}^{\alpha_{1}} L_{2}^{\alpha_{2}-1}[(|\alpha|-2) !]^{s} .
$$

The treatment for the term $\left\|\partial^{\alpha}\left(h_{p}^{2} h_{q q}\right)\right\|_{0}$ is completely the same as above, so we have

$$
\left\|\partial^{\alpha}\left(h_{p}^{2} h_{q q}\right)\right\|_{0} \leq c_{11} L_{1}^{\alpha_{1}} L_{2}^{\alpha_{2}-1}[(|\alpha|-2) !]^{s} .
$$

Combining the above two estimates, we choose $c_{2}=2\left(c_{8}+c_{9}+c_{10}\right)+c_{11}$ to complete the proof of the lemma.

Lemma 4.7. Let $\gamma \in G^{s}\left(\left[p_{0}, 0\right]\right)$. We have, for $\alpha=\left(\alpha_{1}, \alpha_{2}\right)=(m-n, n)$ with $1 \leq n \leq m$,

$$
\left\|\partial^{\alpha}\left(\gamma h_{p}^{3}\right)\right\|_{0} \leq c_{3} L_{1}^{\alpha_{1}-2} L_{2}^{\alpha_{2}}[(|\alpha|-2) !]^{s} .
$$


Proof of the lemma. As for $\left\|\partial^{\xi} h_{q}\right\|_{0}$ in (42), we have by induction

$$
\forall \beta \leq \alpha, \quad|\beta| \geq 3, \quad\left\|\partial^{\beta} h_{p}\right\|_{0} \leq L_{1}^{\beta_{1}-2} L_{2}^{\beta_{2}}[(|\beta|-3) !]^{s} .
$$

Thus using Lemma 4.4-(d) with $H_{i}=L_{i}, i=1,2, u=v=w=h_{p}$, we deduce that $c_{*}^{-1} L_{1} h_{p}^{3} \in \mathcal{A}_{1}$, that is,

$$
\forall \beta \leq \alpha, \quad|\beta| \geq 2, \quad\left\|\partial^{\beta}\left(h_{p}^{3}\right)\right\|_{0} \leq c_{*} L_{1}^{\beta_{1}-2} L_{2}^{\beta_{2}}[(|\beta|-2) !]^{s} .
$$

On the other hand, since $\gamma(p) \in G^{s}\left(\left[p_{0}, 0\right]\right)$, then using (30]) gives

$$
\forall|\beta| \geq 3, \quad\left\|\partial^{\beta} \gamma\right\|_{0} \leq \begin{cases}0, & \beta_{1} \geq 1 \\ \left\|\partial_{p}^{\beta_{2}} \gamma\right\|_{0} \leq M^{\beta_{2}+1}\left(\beta_{2} !\right)^{s} \leq \tilde{M}^{\beta_{2}}\left[\left(\beta_{2}-3\right) !\right]^{s}, & \beta_{1}=0\end{cases}
$$

where $\tilde{M}$ in the last inequality is a constant depending only on $M$ and $s$. Thus if we choose $L_{1}, L_{2}$ in such a way that

$$
L_{2} \geq L_{1} \tilde{M}
$$

then we have

$$
\forall \beta \leq \alpha, \quad|\beta| \geq 3, \quad\left\|\partial^{\beta} \gamma\right\|_{0} \leq L_{1}^{\beta_{1}-2} L_{2}^{\beta_{2}}[(|\beta|-3) !]^{s} .
$$

Now we write

$$
\left\|\partial^{\alpha}\left(\gamma h_{p}^{3}\right)\right\|_{0} \leq \sum_{|\beta| \leq|\alpha|} \frac{|\alpha| !}{|\beta| !(|\alpha|-|\beta|) !}\left\|\partial^{\beta} \gamma\right\|_{0}\left\|\partial^{\alpha-\beta}\left(h_{p}^{3}\right)\right\|_{0}
$$

This together with (46) and (48) allows us to argue as the treatment of $J_{1}-J_{3}$ in Lemma 4.5. to conclude

$$
\left\|\partial^{\alpha}\left(\gamma h_{p}^{3}\right)\right\|_{0} \leq c_{12} L_{1}^{\alpha_{1}-2} L_{2}^{\alpha_{2}}[(|\alpha|-2) !]^{s} .
$$

Thus the desired estimate follows if choosing $c_{3}=c_{12}$. The proof is thus complete.

We now continue the proof of Proposition 4.1. Combining (40) and the conclusions in the previous three lemmas, Lemma 4.5-Lemma 4.7, we get

$$
\begin{aligned}
\left\|\partial_{q}^{m-n} \partial_{p}^{n+2} h\right\|_{0} & \leq\left(\left(c_{1}+c_{3}\right) L_{1}^{-1}+c_{2} L_{1} L_{2}^{-1}\right) L_{1}^{\alpha_{1}-1} L_{2}^{\alpha_{2}}[(|\alpha|-2) !]^{s} \\
& \leq \frac{1}{2} L_{1}^{\alpha_{1}-1} L_{2}^{\alpha_{2}}[(|\alpha|-2) !]^{s}
\end{aligned}
$$

where in the last inequality we chose

$$
L_{1} \geq 4\left(c_{1}+c_{3}\right), \quad L_{2} \geq 4 c_{2} L_{1}
$$

Then we get the desired estimate (38), and thus the validity of $\left(F_{m, n}\right)$ and $\left(F_{m}\right)$. Summarizing the relations (31), (34), (37), (47) and (49), we can choose

$$
L_{1} \geq \max \left\{L,\|h\|_{4}+1,4\left(c_{1}+c_{3}\right)\right\} \text { and } L_{2} \geq\left(8+4 c_{2}+\tilde{M}\right) L_{1},
$$

with $\tilde{M}$ the constant appearing in (47), to complete the proof of Proposition 4.1, 
The rest of this section is devoted to

Proof of Lemma 4.4. To simplify the notations, we use $a_{j}, j \geq 1$, to denote different suitable harmless constants larger than 1, which depend only on the dimension, but are independent of the order $\alpha_{0}$ of derivative.

(a) Assume $u \in \mathcal{A}_{2}$ and $v \in \mathcal{A}_{1}$. By Leibniz formula we have, for any $\alpha \leq \alpha_{0}$ with $|\alpha| \geq 2$,

$$
\partial^{\alpha}(u v)=\sum_{0 \leq \beta \leq \alpha}\left(\begin{array}{c}
\alpha \\
\beta
\end{array}\right)\left(\partial^{\beta} u\right)\left(\partial^{\alpha-\beta} v\right)
$$

Then

$$
\left\|\partial^{\alpha}(u v)\right\| \leq \sum_{0 \leq \beta \leq \alpha} \frac{|\alpha| !}{|\beta| !|\alpha-\beta| !}\left\|\partial^{\beta} u\right\|_{0}\left\|\partial^{\alpha-\beta} v\right\|_{0}=I_{1}+I_{2}+I_{3}
$$

with

$$
\begin{aligned}
& I_{1}=\sum_{\substack{0 \leq \beta \leq \alpha \\
|\beta| \leq 2}} \frac{|\alpha| !}{|\beta| !|\alpha-\beta| !}\left\|\partial^{\beta} u\right\|_{0}\left\|\partial^{\alpha-\beta} v\right\|_{0}, \\
& I_{2}=\sum_{\substack{0 \leq \beta \leq \alpha \\
3 \leq|\beta| \leq|\alpha|-2}} \frac{|\alpha| !}{|\beta| !|\alpha-\beta| !}\left\|\partial^{\beta} u\right\|_{0}\left\|\partial^{\alpha-\beta} v\right\|_{0}, \\
& I_{3}=\sum_{\substack{0 \leq \beta \leq \alpha \\
|\beta| \geq|\alpha|-1}} \frac{|\alpha| !}{|\beta| !|\alpha-\beta| !}\left\|\partial^{\beta} u\right\|_{0}\left\|\partial^{\alpha-\beta} v\right\|_{0} .
\end{aligned}
$$

Since $H_{2} \geq H_{1}$, direct computation shows that there exists $a_{1}>1$ such that

$$
I_{1}+I_{3} \leq a_{1}\left(\|u\|_{2}+\|v\|_{1}+1\right)^{2} H_{1}^{\alpha_{1}-1} H_{2}^{\alpha_{2}}[(|\alpha|-2) !]^{s} .
$$

For $I_{2}$, which appears only when $|\alpha| \geq 5$, we have

$$
\begin{aligned}
I_{2} & \leq \sum_{\substack{0 \leq \beta \leq \alpha \\
3 \leq|\beta| \leq|\alpha|-2}} \frac{|\alpha| !}{|\beta| !|\alpha-\beta| !} H_{1}^{\beta_{1}-2} H_{2}^{\beta_{2}}((|\beta|-3) !)^{s} H_{1}^{\alpha_{1}-\beta_{1}-1} H_{2}^{\alpha_{2}-\beta_{2}}[(|\alpha|-|\beta|-2) !]^{s} \\
& \leq a_{2} \sum_{\substack{0 \leq \beta \leq \alpha \\
3 \leq|\beta| \leq|\alpha|-2}} \frac{|\alpha| !}{|\beta|^{3}|\alpha-\beta|^{2}} H_{1}^{\alpha_{1}-3} H_{2}^{\alpha_{2}}[(|\beta|-3) !]^{s-1}[(|\alpha|-|\beta|-2) !]^{s-1} \\
& \leq a_{2} H_{1}^{\alpha_{1}-3} H_{2}^{\alpha_{2}} \sum_{\substack{0 \leq \beta \leq \alpha \\
3 \leq|\beta| \leq|\alpha|-2}} \frac{|\alpha| !}{|\beta|^{3}|\alpha-\beta|^{2}}[(|\alpha|-5) !]^{s-1} \\
& \leq a_{2} H_{1}^{\alpha_{1}-1} H_{2}^{\alpha_{2}}[(|\alpha|-2) !]^{s} \sum_{\substack{0 \leq \beta \leq \alpha \\
3 \leq|\beta| \leq|\alpha|-2}} \frac{|\alpha|^{2}}{|\beta|^{3}(|\alpha|-|\beta|)^{2}} \\
& \leq a_{3} H_{1}^{\alpha_{1}-1} H_{2}^{\alpha_{2}}[(|\alpha|-2) !]^{s} .
\end{aligned}
$$


In view of the estimates for $I_{1}, I_{2}$ and $I_{3}$, we have, for any $\alpha \leq \alpha_{0}$ with $|\alpha| \geq 2$,

$$
\left\|\partial^{\alpha}(u v)\right\|_{0} \leq a_{4}\left(\|u\|_{2}+\|v\|_{1}+1\right)^{2} H_{1}^{\alpha_{1}-1} H_{2}^{\alpha_{2}}[(|\alpha|-2) !]^{s}
$$

by choosing $a_{4}=\left(a_{1}+a_{3}\right)$.

If additionally $w \in \mathcal{A}_{2}$, then applying the above arguments to the two functions $w$ and

$$
a_{4}^{-1}\left(\|u\|_{2}+\|v\|_{1}+1\right)^{-2} u v
$$

which lies in $\mathcal{A}_{1}$ due to (51), gives

$$
\begin{aligned}
\left\|\partial^{\alpha}(u v w)\right\|_{0} & \leq a_{4}^{2}\left(\|u\|_{2}+\|v\|_{1}+1\right)^{2}\left(\|w\|_{2}+\|u v\|_{1}+1\right)^{2} H_{1}^{\alpha_{1}-1} H_{2}^{\alpha_{2}}[(|\alpha|-2) !]^{s} \\
& \leq a_{4}^{2}\left(\|u\|_{2}+\|v\|_{1}+\|w\|_{2}+1\right)^{6} H_{1}^{\alpha_{1}-1} H_{2}^{\alpha_{2}}[(|\alpha|-2) !]^{s} .
\end{aligned}
$$

Thus the conclusion (国) follows if we choose $c_{*} \geq a_{4}^{2}\left(\|u\|_{2}+\|v\|_{1}+\|w\|_{2}+1\right)^{6}$.

(b) Now assume $u \in \mathcal{A}_{2}$ and $v, w \in \mathcal{A}_{1}$. Firstly note from (51) that

$$
a_{4}^{-1}\left(\|u\|_{2}+\|v\|_{1}+1\right)^{-2} u v \in \mathcal{A}_{1} .
$$

We can use the same arguments as above to the two functions $a_{4}^{-1}\left(\|u\|_{2}+\|v\|_{1}+1\right)^{-2} u v$ and $w$; this gives, for any $\alpha \leq \alpha_{0}$ with $|\alpha| \geq 1$,

$$
\begin{aligned}
a_{4}^{-1}\left(\|u\|_{2}+\|v\|_{1}+1\right)^{-2}\left\|\partial^{\alpha}(u v w)\right\|_{0} \leq & a_{5}\left(\|u v\|_{1}+\|w\|_{1}+1\right)^{2} H_{1}^{\alpha_{1}} H_{2}^{\alpha_{2}}[(|\alpha|-1) !]^{s} \\
& +a_{5} H_{1}^{\alpha_{1}-1} H_{2}^{\alpha_{2}}[(|\alpha|-2) !]^{s} \sum_{\substack{0 \leq \beta \leq \alpha \\
2 \leq|\beta| \leq|\alpha|-2}} \frac{|\alpha|^{2}}{|\beta|^{2}(|\alpha|-|\beta|)^{2}} \\
\leq & a_{6}\left(\|u v\|_{1}+\|w\|_{1}+1\right)^{2} H_{1}^{\alpha_{1}} H_{2}^{\alpha_{2}}[(|\alpha|-1) !]^{s},
\end{aligned}
$$

where the last inequality using the estimate

$$
\sum_{\substack{0 \leq \beta \leq \alpha \\ 2 \leq|\beta| \leq|\alpha|-2}} \frac{|\alpha|^{2}}{|\beta|^{2}(|\alpha|-|\beta|)^{2}} \leq 8 \pi^{2} .
$$

Accordingly,

$$
\left\|\partial^{\alpha}(u v w)\right\|_{0} \leq a_{4} a_{6}\left(\|u\|_{2}+\|v\|_{1}+\|w\|_{1}+1\right)^{6} H_{1}^{\alpha_{1}} H_{2}^{\alpha_{2}}[(|\alpha|-1) !]^{s} .
$$

Thus the conclusion follows if we choose $c_{*} \geq a_{4} a_{6}\left(\|u\|_{2}+\|v\|_{1}+\|w\|_{1}+1\right)^{6}$.

(IC) Now consider the case when $u, v \in \mathcal{A}_{2}$ and $w \in \mathcal{A}_{0}$. Similarly we can first use the same arguments as in (国), to obtain

$$
a_{7}^{-1}\left(\|u\|_{2}+\|w\|_{0}+1\right)^{-2} u w \in \mathcal{A}_{0},
$$

and then repeat the arguments to the two functions $a_{7}^{-1}\left(\|u\|_{2}+\|w\|_{0}+1\right)^{-2} u w$ and $v$ to conclude

$$
a_{7}^{-1} a_{8}^{-1}\left(\|u\|_{2}+\|v\|_{2}+\|w\|_{0}+1\right)^{-6} u v w \in \mathcal{A}_{0} .
$$


The conclusion (두) follows by choosing $c_{*} \geq a_{7} a_{8}\left(\|u\|_{2}+\|v\|_{2}+\|w\|_{0}+1\right)^{6}$.

(d) Assume $u, v, w \in \mathcal{A}_{2}$. Similarly, we can first argue as in (a) to show that

$$
\forall|\alpha| \geq 2, \quad\left\|\partial^{\alpha}(u v)\right\|_{0} \leq a_{9}\left(\|u\|_{2}+\|v\|_{2}+1\right)^{2} H_{1}^{\alpha_{1}-2} H_{2}^{\alpha_{2}}[(|\alpha|-2) !]^{s},
$$

and then repeat the arguments to $a_{9}^{-1}\left(\|u\|_{2}+\|v\|_{2}+1\right)^{-2} u v$ and $w$ to derive

$$
\forall|\alpha| \geq 2, \quad\left\|\partial^{\alpha}(u v w)\right\|_{0} \leq a_{9} a_{10}\left(\|u\|_{2}+\|v\|_{2}+\|w\|_{2}+1\right)^{6} H_{1}^{\alpha_{1}-2} H_{2}^{\alpha_{2}}[(|\alpha|-2) !]^{s} .
$$

Thus the conclusion (d) follows by choosing $c_{*} \geq a_{9} a_{10}\left(\|u\|_{2}+\|v\|_{2}+\|w\|_{2}+1\right)^{6}$.

Finally, the conclusion of Lemma 4.4 follows by choosing

$$
c_{*} \geq\left(a_{4}^{2}+a_{4} a_{6}+a_{7} a_{8}+a_{9} a_{10}\right)\left(\|u\|_{2}+\|v\|_{2}+\|w\|_{2}+1\right)^{6}
$$

with $a_{j}$ the constants depending only on the dimension. The proof is thus complete.

\section{Regularity of water waves with surface tension}

Adding the effects of surface tension in the free boundary problem (3a $)-(3 \mathrm{~d})$ introduces higher-order derivative into the boundary condition. That is the equation (3b) is replaced by

$$
|\nabla \psi|^{2}+2 g(y+d)-2 \sigma \frac{\eta_{x x}}{\left(1+\eta_{x}^{2}\right)^{\frac{3}{2}}}=Q, \quad y=\eta(x),
$$

where $\sigma>0$ is the coefficient of surface tension. Correspondingly, the equation (4b) becomes

$$
1+h_{q}^{2}+(2 g h-Q) h_{p}^{2}-2 \sigma \frac{h_{p}^{2} h_{q q}}{\left(1+h_{q}^{2}\right)^{\frac{3}{2}}}=0, \quad \text { on } \quad p=0 .
$$

Proven in this section is the regularity property of all the streamlines and stream function of water waves with surface tension.

Theorem 5.1. Consider the free boundary problem (3a)-(3d) with (3b) replaced by above $\left(3 b^{\prime}\right)$. Suppose $\gamma \in C^{0, \mu}\left(\left[p_{0}, 0\right]\right)$ with $\mu$ and $p_{0}$ given. Then each streamline including the free surface $y=\eta(x)$ is a real-analytic curve. If, in addition, $\gamma \in G^{s}\left(\left[p_{0}, 0\right]\right)$ with $s \geq 1$, then $\psi(x, y) \in G^{s}(\bar{\Omega})$; in particular if $s=1$, i.e., $\gamma$ is analytic in $\left[p_{0}, 0\right]$, then the stream function $\psi(x, y)$ is analytic in $\bar{\Omega}$.

Proof. As before we only prove the corresponding regularity for height function $h$ of the system (4a)-(4c) with (4b) replaced by above (4b'). Since the arguments are nearly the same as those in the absence of surface tension (Section 3 and Section 4), we shall only give a sketch and indicate how to modify the analysis as adding the higher-order derivative due to surface tension.

Repeating the arguments in Section 4, we can derive the second statement in Theorem 5.1, without any difference. So we only need to prove the first statement on the 
analyticity of streamlines, where the main difference from Section 3 occurs. As in Remark 3.3 we may assume $\partial_{q}^{k} h \in C^{2, \mu}(\bar{R})$ for any $k \in \mathbb{N}$. Taking $m^{t h}$-order derivative with respect to the $q$-variable on both sides of the equation (4b') shows that the second equation in (10) becomes

$$
\tilde{B}(h)\left[\partial_{q}^{m} h\right]=\tilde{\varphi}_{1}+\tilde{\varphi}_{2}, \quad \text { on } p=0
$$

with the operator

$$
\tilde{B}(h)=2 \sigma \frac{h_{p}^{2}}{\left(1+h_{q}^{2}\right)^{\frac{3}{2}}} \partial_{q}^{2}
$$

and the right-hand side

$$
\tilde{\varphi}_{1}=\partial_{q}^{m} h_{q}^{2}+\partial_{q}^{m}\left((2 g h-Q) h_{p}^{2}\right), \quad \tilde{\varphi}_{2}=-2 \sigma \sum_{1 \leq n \leq m}\left(\partial_{q}^{m-n} h_{q q}\right)\left(\partial_{q}^{n} \frac{h_{p}^{2}}{\left(1+h_{q}^{2}\right)^{\frac{3}{2}}}\right) .
$$

The first and third equations in (10) remain unchanged. Then, as before, our aim is to show that the corresponding estimate as in Proposition 3.1 holds, that is, there exists a constant $\tilde{L} \geq 1$ such that for any $m \geq 2$,

$$
\left(\tilde{E}_{m}\right): \quad\left\|\partial_{q}^{m} h\right\|_{2, \mu} \leq \tilde{L}^{m-1}(m-2) !
$$

and to this end the main point is to show that $\left(\tilde{E}_{m}\right)$ holds under the assumption that for any $j$ with $2 \leq j \leq m-1$, the following estimate

$$
\left(\tilde{E}_{j}\right): \quad\left\|\partial_{q}^{j} h\right\|_{2, \mu} \leq L^{j-1}(j-2) !
$$

is already valid.

Since $h \in C^{2, \mu}(\bar{R})$, the coefficient $\frac{h_{p}^{2}}{\left(1+h_{q}^{2}\right)^{\frac{3}{2}}}$ of the operator $\tilde{B}(h)$ is in $C^{1, \mu}(\bar{R})$. Moreover by the induction assumption (52), $\tilde{\varphi}_{1}$ and $\tilde{\varphi}_{2}$ are in $C^{0, \mu}(\bar{R})$. Furthermore it has been shown in [10] that the operator $\tilde{B}(h)$ satisfies the complementing condition in the sense of [1]. As a result, we can apply the Schauder estimate in [1] to conclude

$$
\left\|\partial_{q}^{m} h\right\|_{2, \mu} \leq \tilde{\mathcal{C}}\left(\left\|\partial_{q}^{m} h\right\|_{0}+\sum_{i=1}^{2}\left\|f_{i}\right\|_{0, \mu}+\left\|\tilde{\varphi}_{1}\right\|_{0, \mu}+\left\|\tilde{\varphi}_{2}\right\|_{0, \mu}\right)
$$

with $\tilde{\mathcal{C}}$ a constant independent of $m$, and $f_{i}, i=1,2$, defined in (11)-(12). As for the first three terms on the right hand side, we can use the similar arguments as in Section 3 without any additional difficulty, to conclude

$$
\left\|\partial_{q}^{m} h\right\|_{0}+\sum_{i=1}^{2}\left\|f_{i}\right\|_{0, \mu}+\left\|\tilde{\varphi}_{1}\right\|_{0, \mu} \leq \tilde{C}_{1} \tilde{L}^{m-2}(m-2) !
$$

with $\tilde{C}_{1}$ a constant independent of $m$. It remains to estimate $\left\|\tilde{\varphi}_{2}\right\|_{0, \mu}$, and show that for some constant $\tilde{C}_{2}$,

$$
\left\|\tilde{\varphi}_{2}\right\|_{0, \mu} \leq \tilde{C}_{2} \tilde{L}^{m-2}(m-2) !
$$

To do so we need the following lemma, whose proof is postponed to the end of this section. 
Lemma 5.2. Let $C_{*} \geq 1$ be the constant given in Lemma 3.4, and let $k_{0} \in \mathbb{N}$ with $k_{0} \geq 3$. Suppose $\partial_{q}^{k} u \in C^{0, \mu}(\bar{R})$ for any $k \leq k_{0}$. If there exist two constants $C_{0}$ and $\tilde{H}$ satisfying

$$
C_{0} \geq C_{*}\left(2\left\|\left(1+u^{2}\right)^{-1}\right\|_{2, \mu}+2\left\|\left(1+u^{2}\right)^{-3 / 2}\right\|_{2, \mu}+\left\|\partial_{q}\left(u^{2}\right)\right\|_{2, \mu}+1\right)^{6}
$$

and

$$
\tilde{H} \geq 2 C_{0}^{2}+\left\|\partial_{q}^{3}\left(\left(1+u^{2}\right)^{-1}\right)\right\|_{0, \mu}+\left\|\partial_{q}^{3}\left(\left(1+u^{2}\right)^{-3 / 2}\right)\right\|_{0, \mu},
$$

such that

$$
\forall 3 \leq k \leq k_{0}, \quad\left\|\partial_{q}^{k}\left(u^{2}\right)\right\|_{0, \mu} \leq C_{0} \tilde{H}^{k-2}(k-3) !
$$

then

$$
\forall 3 \leq k \leq k_{0}, \quad\left\|\partial_{q}^{k}\left(\left(1+u^{2}\right)^{-3 / 2}\right)\right\|_{0, \mu} \leq C_{0}^{2} \tilde{H}^{k-2}(k-3) !
$$

We now use the above lemma to prove (54). Observe

$$
\left\|\tilde{\varphi}_{2}\right\|_{0, \mu} \leq 2 \sigma \sum_{1 \leq n \leq m}\left\|\partial_{q}^{m-n} h_{q q}\right\|_{0, \mu}\left\|\partial_{q}^{n}\left(h_{p}^{2}\left(1+h_{q}^{2}\right)^{-3 / 2}\right)\right\|_{0, \mu} .
$$

Using the induction assumption (52), we have

$$
\forall 1 \leq n \leq m-2, \quad\left\|\partial_{q}^{m-n} h_{q q}\right\|_{0, \mu} \leq\left\|\partial_{q}^{m-n} h\right\|_{2, \mu} \leq \tilde{L}^{m-n-1}(m-n-2) !,
$$

and

$$
\forall 3 \leq n \leq m, \quad\left\|\partial_{q}^{n} h_{q}\right\|_{0, \mu} \leq\left\|\partial_{q}^{n-1} h\right\|_{2, \mu} \leq \tilde{L}^{n-2}(n-3) ! .
$$

This last inequality along with Lemma 3.4, with $u_{1}=u_{2}=h_{q}, u_{3}=1$, implies

$$
\forall 3 \leq n \leq m, \quad\left\|\partial_{q}^{n} h_{q}^{2}\right\|_{0, \mu} \leq C_{*}\left(2\left\|h_{q}\right\|_{3, \mu}+1\right)^{6} \tilde{L}^{n-2}(n-3) ! \leq C_{0} \tilde{L}^{n-2}(n-3) !,
$$

where in the last inequality we choose

$$
C_{0} \geq C_{*}\left(2\left\|\left(1+h_{q}^{2}\right)^{-1}\right\|_{2, \mu}+2\left\|\left(1+h_{q}^{2}\right)^{-3 / 2}\right\|_{2, \mu}+\left\|\partial_{q}\left(h_{q}^{2}\right)\right\|_{2, \mu}+2\left\|h_{q}\right\|_{3, \mu}+1\right)^{6} .
$$

Now choosing $\tilde{L}$ in such a way that

$$
\tilde{L} \geq 2 C_{0}^{2}+\left\|\partial_{q}^{3}\left(\left(1+h_{q}^{2}\right)^{-1}\right)\right\|_{0, \mu}+\left\|\partial_{q}^{3}\left(\left(1+h_{q}^{2}\right)^{-3 / 2}\right)\right\|_{0, \mu},
$$

then applying the above Lemma 5.2 , with $k_{0}=m, u=h_{q}$ and $\tilde{H}=\tilde{L}$, we have,

$$
\forall 3 \leq n \leq m, \quad\left\|\partial_{q}^{n}\left(\left(1+h_{q}^{2}\right)^{-3 / 2}\right)\right\|_{0, \mu} \leq C_{0}^{2} \tilde{L}^{n-2}(n-3) ! .
$$

This along with (59) allows us to argue as in the proof of (17) for $\left\|f_{1}\right\|_{0, \mu}$, to obtain (54). Thus choosing

$$
\tilde{L} \geq \tilde{\mathcal{C}}\left(\tilde{C}_{1}+\tilde{C}_{2}\right)+2 C_{0}^{2}+\left\|\partial_{q}^{3}\left(\left(1+h_{q}^{2}\right)^{-1}\right)\right\|_{0, \mu}+\left\|\partial_{q}^{3}\left(\left(1+h_{q}^{2}\right)^{-3 / 2}\right)\right\|_{0, \mu},
$$

we get the validity of $\left(\tilde{E}_{m}\right)$. The proof of Theorem 5.1 is thus complete. 
The rest is occupied by

Proof of Lemma 5.2. As a preliminary step we first use induction to prove

$$
\forall 3 \leq k \leq k_{0}, \quad\left\|\partial_{q}^{k}\left(\left(1+u^{2}\right)^{-1}\right)\right\|_{0, \mu} \leq \tilde{H}^{k-1}(k-2) ! .
$$

In fact (60) obviously holds when $k=3$ due to (56). Now assuming $k \geq 4$ and that

$$
\forall 3 \leq j \leq k-1, \quad\left\|\partial_{q}^{j}\left(\left(1+u^{2}\right)^{-1}\right)\right\|_{0, \mu} \leq \tilde{H}^{j-1}(j-2) !,
$$

we show that the above inequality still holds for $k$ with $k \leq k_{0}$. To do so, write

$$
\partial_{q}^{k}\left(\left(1+u^{2}\right)^{-1}\right)=-\partial_{q}^{k-1}\left(\left(1+u^{2}\right)^{-1}\left(1+u^{2}\right)^{-1} \partial_{q}\left(u^{2}\right)\right) \text {. }
$$

Next we intend to apply Lemma 3.4 to prove that the right-hand side of the above equation satisfies

$$
\left\|\partial_{q}^{k-1}\left(\left(1+u^{2}\right)^{-1}\left(1+u^{2}\right)^{-1} \partial_{q}\left(u^{2}\right)\right)\right\|_{0, \mu} \leq C_{0}^{2} \tilde{H}^{k-2}(k-3) !
$$

In fact for any $j$ with $3 \leq j \leq k-1$, one has by (157),

$$
\left\|\partial_{q}^{j} \partial_{q}\left(u^{2}\right)\right\|_{0, \mu} \leq C_{0} \tilde{H}^{j-1}(j-2) !
$$

and by the induction assumption (61)

$$
\left\|\partial_{q}^{j}\left(\left(1+u^{2}\right)^{-1}\right)\right\|_{0, \mu} \leq \tilde{H}^{j-1}(j-2) !
$$

The above two estimates allow us to use Lemma 3.4, with $\ell=1, u_{1}=u_{2}=\left(1+u^{2}\right)^{-1}$ and $u_{3}=C_{0}^{-1} \partial_{q}\left(u^{2}\right)$, to obtain

$$
\begin{aligned}
& \left\|\partial_{q}^{k-1}\left(\left(1+u^{2}\right)^{-1}\left(1+u^{2}\right)^{-1} \partial_{q}\left(u^{2}\right)\right)\right\|_{0, \mu} \\
\leq & C_{0} C_{*}\left(2\left\|\left(1+u^{2}\right)^{-1}\right\|_{2, \mu}+C_{0}^{-1}\left\|\partial_{q}\left(u^{2}\right)\right\|_{2, \mu}+1\right)^{6} \tilde{H}^{k-2}(k-3) ! \\
\leq & C_{0}^{2} \tilde{H}^{k-2}(k-3) !
\end{aligned}
$$

the last inequality using (55). Thus the desired inequality (63) follows. As a result, combining (62) and (63), we conclude

$$
\left\|\partial_{q}^{k}\left(\left(1+u^{2}\right)^{-1}\right)\right\|_{0, \mu} \leq C_{0}^{2} \tilde{H}^{k-2}(k-3) ! \leq \tilde{H}^{k-1}(k-2) !
$$

where the last inequality holds because $\tilde{H} \geq 2 C_{0}^{2}$ due to (56). We have proven (60).

Now we prove (58), which obviously holds when $k=3$ in view of (56). Now assuming $k \geq 4$ and that

$$
\forall 3 \leq j \leq k-1, \quad\left\|\partial_{q}^{j}\left(\left(1+u^{2}\right)^{-3 / 2}\right)\right\|_{0, \mu} \leq C_{0}^{2} \tilde{H}^{j-2}(j-3) !,
$$


we show the above equality still holds for $k$ with $k \leq k_{0}$. As before, write

$$
\partial_{q}^{k}\left(\left(1+u^{2}\right)^{-3 / 2}\right)=-\partial_{q}^{k-1}\left(\frac{3\left(1+u^{2}\right)^{-3 / 2}}{2}\left(1+u^{2}\right)^{-1} \partial_{q}\left(u^{2}\right)\right) .
$$

Then the estimate in (64) for $j=k$ will hold if we can show that

$$
\frac{3}{2}\left\|\partial_{q}^{k-1}\left(\left(1+u^{2}\right)^{-3 / 2}\left(1+u^{2}\right)^{-1} \partial_{q}\left(u^{2}\right)\right)\right\|_{0, \mu} \leq C_{0}^{2} \tilde{H}^{k-2}(k-3) ! .
$$

Again we next intend to apply Lemma 3.4 to prove the above estimate . For any $j$ with $3 \leq j \leq k-1$, one has by (57)

$$
\left\|\partial_{q}^{j} \partial_{q}\left(u^{2}\right)\right\|_{0, \mu} \leq C_{0} \tilde{H}^{j-1}(j-2) !
$$

and by the induction assumption (64)

$$
\frac{3}{2}\left\|\partial_{q}^{j}\left(\left(1+u^{2}\right)^{-3 / 2}\right)\right\|_{0, \mu} \leq \frac{3}{2} C_{0}^{2} \tilde{H}^{j-2}(j-3) ! \leq \tilde{H}^{j-1}(j-2) !,
$$

the last inequality using the fact that $\tilde{H} \geq 2 C_{0}^{2} \geq 3 C_{0}^{2} / 2$ due to (56). The above two estimates along with (60) allow us to use Lemma 3.4, with $\ell=1$,

$$
u_{1}=\frac{3}{2}\left(1+u^{2}\right)^{-3 / 2}, \quad u_{2}=\left(1+u^{2}\right)^{-1}, \quad u_{3}=C_{0}^{-1} \partial_{q}\left(u^{2}\right)
$$

this gives

$$
\begin{aligned}
& \frac{3}{2}\left\|\partial_{q}^{k-1}\left(\left(1+u^{2}\right)^{-3 / 2}\left(1+u^{2}\right)^{-1} \partial_{q}\left(u^{2}\right)\right)\right\|_{0, \mu} \\
\leq & C_{0} C_{*}\left(\frac{3}{2}\left\|\left(1+u^{2}\right)^{-3 / 2}\right\|_{2, \mu}+\left\|\left(1+u^{2}\right)^{-1}\right\|_{2, \mu}+\left\|\partial_{q}\left(u^{2}\right)\right\|_{2, \mu}+1\right)^{6} \tilde{H}^{k-2}(k-3) ! \\
\leq & C_{0}^{2} \tilde{H}^{k-2}(k-3) !,
\end{aligned}
$$

where the last inequality holds because of (55). Thus the desired estimate (66) follows. We have proven (58), completing the proof of Lemma 5.2 .

Acknowledgments The work is supported by NSFC(11001207,11201355) and the RFDP (20100141120064).

\section{References}

[1] S. Agmon, A. Douglis, and L. Nirenberg. Estimates near the boundary for solutions of elliptic partial differential equations satisfying general boundary conditions. I. Comm. Pure Appl. Math., 12:623-727, 1959.

[2] Hua Chen, Wei-Xi Li and Chao-Jiang Xu. Gevrey regularity of subelliptic MongeAmpère equations in the plan. Advances in Mathematics , 228:1816-1841, 2011. 
[3] Adrian Constantin and Joachim Escher. Analyticity of periodic traveling free surface water waves with vorticity. Ann. of Math. (2), 173(1):559-568, 2011.

[4] Adrian Constantin and Walter Strauss. Exact steady periodic water waves with vorticity. Comm. Pure Appl. Math., 57(4):481-527, 2004.

[5] David Gilbarg and Neil S. Trudinger. Elliptic partial differential equations of second order. Classics in Mathematics. Springer-Verlag, Berlin, 2001. Reprint of the 1998 edition.

[6] M. D. Groves and E. Wahlén. Spatial dynamics methods for solitary gravity-capillary water waves with an arbitrary distribution of vorticity. SIAM J. Math. Anal., 39(3):932-964, 2007.

[7] M. D. Groves and E. Wahlén. Small-amplitude Stokes and solitary gravity water waves with an arbitrary distribution of vorticity. Phys. D, 237(10-12):1530-1538, 2008.

[8] David Henry. Analyticity of the streamlines for periodic travelling free surface capillary-gravity water waves with vorticity. SIAM J. Math. Anal., 42(6):3103-3111, 2010.

[9] David Henry. On the regularity of capillary water waves with vorticity. C. R. Math. Acad. Sci. Paris, 349(3-4):171-173, 2011.

[10] David Henry. Analyticity of the Free Surface for Periodic Travelling CapillaryGravity Water Waves with Vorticity. Journal of Math. Fluid Mech. , 2011, DOI: 10.1007/s00021-011-0056-z.

[11] Vera Mikyoung Hur. Global bifurcation theory of deep-water waves with vorticity. SIAM J. Math. Anal., 37(5):1482-1521 (electronic), 2006.

[12] Vera Mikyoung Hur. Exact solitary water waves with vorticity. Arch. Ration. Mech. Anal., 188(2):213-244, 2008.

[13] Vera Mikyoung Hur. Stokes waves with vorticity. J. Anal. Math., 113:331-386, 2011.

[14] Vera Mikyoung Hur. Analyticity of Rotational Flows Beneath Solitary Water Waves. Int Math Res Notices 2011., doi: 10.1093/imrn/rnr123.

[15] D. Kinderlehrer, L. Nirenberg, and J. Spruck. Regularity in elliptic free boundary problems. J. Analyse Math., 34:86-119 (1979), 1978.

[16] Hans Lewy. A note on harmonic functions and a hydrodynamical application. Proc. Amer. Math. Soc., 3:111-113, 1952. 
[17] James Lighthill. Waves in fluids. Cambridge Mathematical Library. Cambridge University Press, Cambridge, 2001. Reprint of the 1978 original.

[18] B.-V. Matioc. Analyticity of the Streamlines for Periodic Traveling Water Waves with Bounded Vorticity, Int Math Res Notices, 17:3858-3871, 2011. doi: 10.1093/imrn/rnq235

[19] B.-V. Matioc. On the regularity of deep-water waves with general vorticity distributions. to appear in Quarterly of Applied Mathematics, 2011.

[20] Luigi Rodino. Linear partial differential operators in Gevrey spaces. World Scientific Publishing Co. Inc., River Edge, NJ, 1993.

[21] Erik Wahlén. Steady periodic capillary-gravity waves with vorticity. SIAM J. Math. Anal., 38(3):921-943 (electronic), 2006. 